\title{
Propofol Attenuates Lung but not Brain Cancer Cell Malignancy through Metabolism and Cell Signaling Modulation In Vitro
}

\section{Cong $\mathrm{Hu}$}

Wenzhou Medical University Second Affiliated Hospital

\section{Masae Iwasaki}

Imperial College London

Zhigang Liu

Imperial College London

\section{Bincheng Wang}

Imperial College London

Xiaomeng Li

Imperial College London

\section{Han Lin}

Wenzhou Medical University Second Affiliated Hospital

Jun Li

Wenzhou Medical University Second Affiliated Hospital

Jia V. Li

Imperial College London

Qingquan Lian ( $\square$ wzmzlqq@163.com )

Wenzhou Medical University Second Affiliated Hospital

Daqing Ma ( $\nabla$ d.ma@imperial.ac.uk)

Imperial College London Faculty of Medicine https://orcid.org/0000-0002-0688-2097

\section{Research}

Keywords: Intravenous anesthetic, lung cancer, neuroglioma, metabolism, PEDF, HIF-1a

Posted Date: July 23rd, 2020

DOI: https://doi.org/10.21203/rs.3.rs-44983/v1

License: (a) (i) This work is licensed under a Creative Commons Attribution 4.0 International License.

Read Full License 


\section{Propofol Attenuates Lung but not Brain Cancer Cell Malignancy through}

\section{Metabolism and Cell Signaling Modulation In Vitro}

3

4 Cong $\mathrm{Hu}^{1,2}$, Masae Iwasaki ${ }^{2,3}$, Zhigang $\mathrm{Liu}^{4}$, Bincheng Wang ${ }^{2}$, Xiaomeng $\mathrm{Li}^{2}$, Han Lin ${ }^{1}$,

5 Jun $\mathrm{Li}^{1}$, Jia V. $\mathrm{Li}^{4}$, Qingquan $\mathrm{Lian}^{1, *}$, Daqing $\mathrm{Ma}^{2, *}$

6

$7 \quad{ }^{1}$ Department of Anesthesiology, The Second Affiliated Hospital and Yuying Children's

8 Hospital of Wenzhou Medical University, Wenzhou, Zhejiang, China

9 2Division of Anaesthetics, Pain Medicine and Intensive Care, Department of Surgery

10 and Cancer, Faculty of Medicine, Imperial College London, Chelsea \& Westminster

11 Hospital, United Kingdom

$12{ }^{3}$ Department of Anesthesiology and Pain Medicine, Graduate School of Medicine,

13 Nippon Medical School, Tokyo, Japan

$14{ }^{4}$ Department of Metabolism, Digestion and Reproduction, Faculty of Medicine, Imperial 15 College London, United Kingdom

$17{ }^{*}$ Correspondence: D Ma, Department of Surgery and Cancer, Faculty of Medicine, 18 Imperial College London, Chelsea \& Westminster Hospital, SW10 9NH, London, 19 United Kingdom, Tel: +44 203315 8495, E-mail: d.ma@imperial.ac.uk

21 Or

22 QQ Lian, Department of Anesthesiology, The $2^{\text {nd }}$ Affiliated Hospital and Yuying 23 Children's Hospital of Wenzhou Medical University, 325027, Wenzhou, Zhejiang, P. R.

24 China, Email: wzmzlqq@163.com

QL, JVL and DM share the senior authorship. 


\section{Abstract}

29 Background: Intravenous anesthesia with propofol was reported to improve cancer

30 surgical outcomes when compared with inhalational anesthesia. However, the

31 underlying molecular mechanisms largely remain unknown. The current study aims to

32 investigate whether propofol affects cancer cell biology including tumor metastasis-

33 related gene expression, cellular signaling and metabolic changes in lung and brain

34 cancer cells.

35 Methods: Lung cancer (A549) or neuroglioma (H4) cells were treated with propofol at

36 a clinically relevant concentration $(4 \mu \mathrm{g} / \mathrm{mL})$ for 2 hours, followed by 24 hours recovery.

37 Tumor metastasis-related gene expressions were assessed using a PCR array and

38 validated with qRT-PCR. Glucose transporter 1 (GLUT1), brain protein 44-like

39 (BRP44L), pigment epithelium-derived factor (PEDF), Akt, phospho-Akt (p-Akt),

40 extracellular-signal-regulated kinase 1/2 (Erk1/2), phospho-Erk1/2 (p-Erk1/2), and

41 hypoxia-inducible factor 1 alpha (HIF-1 $\alpha$ ) expressions were determined using

42 immunofluorescent staining and/or western blotting. The metabolites in cell extract and

43 media following propofol treatment were characterized using proton nuclear magnetic

44 resonance $\left({ }^{1} \mathrm{H}\right.$ NMR) spectroscopy. The malignant hallmarks including cell viability,

45 proliferation, migration, and invasion were evaluated using cell counting kit-8 (CCK-8)

46 assay, Ki-67 staining, wound healing and transwell assay, respectively.

47 Results: Propofol reduced cell viability and inhibited cell proliferation, migration and 48 invasion of lung cancer cells, but not neuroglioma cells. In lung cancer cells, gene 
50 downregulated, while NR4A3, RB1, NME1, MTSS1, NME4, SYK, APC, and FAT1 were

51 upregulated following the propofol treatment. Furthermore, propofol downregulated

52 GLUT1, BRP44L, p-Akt, p-Erk, and HIF-1a expressions in lung cancer cells and

53 upregulated PEDF expression. Propofol increased glutamate and glycine but

54 decreased acetate and formate in lung cancer cells whilst increased lactate, valine,

55 isoleucine, and leucine and glycerol, and decreased pyruvate and isopropanol in the

56 culture media. Consistent with the phenotypical changes, these molecular and

57 metabolic changes were not observed in the neuroglioma cells.

58 Conclusions: Our findings indicated "anti-tumor" effects of propofol on the lung cancer

59 but not neuroglioma, through the regulation of tumor metastasis-related genes, multi-

60 cellular signaling and cellular metabolism.

61

62 Keywords: Intravenous anesthetic; lung cancer; neuroglioma; metabolism; PEDF;

63 HIF-1 $1 \alpha$.

64

65

66

67

68

69 


\section{Background}

71 Cancer is the second leading disease of death worldwide $(1,2)$. Owing to ageing

72 population globally, the incidence of cancer is increasing (2). Among all the types of

73 cancers, lung cancer caused the highest mortality in adults reported in 2017 (3), whilst

74 in children, the mortality of brain tumor is higher than other cancer types (4).

75

76 Surgery remains the primary therapy for solid organ cancer including lung cancer (5,

6). However, most cancer patients after surgery die due to the metastasis and recurrence. Recurrence can be the local, regional and distant recurrence (7) and the recurrence type, severity, and incidence are determined by many factors, including malignancy of cancer, surgical trauma and stress, adjuvant therapies and beyond (8).

82 It has been recognized that anesthetics and techniques may also contribute to the

83 outcomes of cancer patients after surgery. For example, it was found that patients

84 received inhalational anesthetics had a higher mortality rate when compared to those

85 who were administered with intravenous anesthetic propofol during their surgical

86 treatments for cancer (9). However, the mechanisms underlying these clinical

87 observations remain unknown. A previous study demonstrated volatile anesthetics,

88 such as, sevoflurane, isoflurane, and desflurane, upregulated the metastatic genes in

89 ovarian cancer cells (10). Furthermore, unlike propofol, isoflurane was also

90 demonstrated to increase hypoxia-inducible factor-1 alpha (HIF-1 $\alpha)(11)$, which is a 
91 transcriptional activator associated with the progression of a variety of cancer types,

92 such as breast, colon, and lung cancer (12). HIF-1 $\alpha$ can be activated by its upstream

93 effectors, such as Akt and Erk1/2 $(13,14)$. Akt, also known as protein kinase B, belongs

94 to the cAMP-dependent protein kinase superfamily, which is involved in many

95 biological functions, for example, cell cycle, nutrient metabolism, and transcriptional

96 regulation (15). Erk1/2 is also involved in a variety of biological functions, including

97 proliferation, differentiation and cell survival (16). Akt and Erk1/2 signaling pathways

98 can be regulated by a diversity of factors, such as pigment epithelium-derived factor

99 (PEDF) $(17,18)$. PEDF is a secreted protein of serine protease inhibitor family and has

100 anti-angiogenic, anti-tumor, and neurotrophic functions; and its therapeutic value for

101 heart disease, choroidal neovascularization and cancer has been explored $(19,20)$.

102

103 In the current study, the role of PEDF and HIF-1 $\alpha$ in anti-cancer property of propofol

104 will be determined in lung and brain cancer cell cultures. We hypothesized that propofol

105 downregulates glucose transporter 1 (GLUT1) and mitochondrial pyruvate carrier 1

106 (MPC1, also called BRP44L, brain protein 44-like) expressions, which leads to the

107 disturbance of metabolisms of cancers. These changes may alter PEDF expression,

108 which in turn, downregulates HIF-1 $\alpha$ expression via Akt and Erk1/2 cellular signaling

109 pathways. The suppression of HIF-1a expression finally affects tumor metastasis-

110 related gene expressions and changes cancer cell malignancy and biology. 


\section{Methods}

\section{Cell culture}

114 Lung cancer (A549) and neuroglioma (H4) cell lines were obtained from ECACC

115 (Wiltshire, UK). A549 cell line was grown in Gibco RPMI media 1640 (ThermoFisher,

116 Paisley, UK) supplemented with 10\% fetal bovine serum (FBS) and $1 \%$ penicillin-

117 streptomycin (ThermoFisher), while $\mathrm{H} 4$ cell line was cultured in Gibco Dulbecco's

118 Modified Eagle Medium (ThermoFisher) supplemented with 10\% FBS and 1\%

119 penicillin-streptomycin. $\mathrm{A} 549$ and $\mathrm{H} 4$ cells were cultured at $37^{\circ} \mathrm{C}$ with $5 \% \mathrm{CO}_{2}$ and

120 balanced with air. Cells were treated with $4 \mu \mathrm{g} / \mathrm{mL}$ propofol (Sigma-Aldrich, Dorset, UK)

121 for 2 hours when the seeded cells formed a continuous monolayer. Intralipid (Santa

122 Cruz Biotechnology, Dallas, Texas, USA) was used as vehicle control. After which, cell

123 medium was replaced with fresh medium for the next 24 hours for further experiments.

\section{Immunofluorescent staining}

126 A549 and H4 cells were fixed with 4\% paraformaldehyde solution in PBS (Santa Cruz

127 Biotechnology) for 15 minutes, which was followed by the blocking procedure with $10 \%$

128 normal donkey serum (Sigma-Aldrich) for 30 minutes. After blocking, they were

129 incubated with the primary antibodies (Supplemental Table 1) at $4^{\circ} \mathrm{C}$ overnight and

130 then probed with the secondary antibodies (Supplemental Table 1). The cells were

131 mounted with DAPI (4', 6-diamidino-2-phenylindole) mounting media (Vector

132 Laboratories, Burlingame, California, USA), and imaged by fluorescent microscopy. 
133 ImageJ 2.0 software (National Institutes of Health, Bethesda, Maryland, USA) was

134 used to quantify analysis wherever necessary.

\section{Western blotting}

137 Western blotting was done using our established protocol (10). The treated cells were

138 lysed in cell lysis buffer (Cell Signaling, Danvers, Massachusetts, USA) and the protein

139 concentration of the supernatant sample of cell lysis was measured. Protein samples

140 with $60 \mu \mathrm{g}$ were loaded into $4-12 \%$ polyacrylamide gel (Life Technologies, Paisley, UK)

141 to be electrophoresed for 1.5 hour. After transferring the protein bands onto a PVDF

142 membrane, the membrane was blocked with $5 \%$ non-fat milk for 1 hour, before

143 incubation with the primary antibodies (Supplemental Table 1) at $4^{\circ} \mathrm{C}$ overnight. After

144 washing, the membrane was incubated with the secondary antibodies (Supplemental

145 Table 1) for 1 hour at room temperature. The membrane was immersed with the

146 enhanced chemiluminescence (ECL) system (Santa Cruz Biotechnology, Dallas,

147 Texas, USA), and developed by GeneSnap (Syngene, Cambridge, UK). The intensity

148 of blot bands was quantified with ImageJ 2.0.

150 Nuclear magnetic resonance spectroscopy

151 Cell pellets were placed into a bead beater tube (STARLAB Science Laboratory,

152 Hamburg, Germany) containing $0.1 \mathrm{~g}$ sterile beads with a diameter of $0.1 \mathrm{~mm}$ and 1.5

$153 \mathrm{~mL}$ of the pre-chilled mixture of methanol (Thermo Fisher) and water $\left(\mathrm{MeOH}: \mathrm{H}_{2} \mathrm{O}, \mathrm{v}: \mathrm{v}\right.$, 
154 1:1). The tubes were placed in a bead beater (Bertin Instruments, Montigny-le-

155 Bretonneux, France) to homogenize the samples using two cycles of $6,500 \mathrm{~Hz}$ for 40

$156 \mathrm{~s}$ with $5 \mathrm{~min}$ on dry ice between cycles. The samples were then centrifuged at 10,000

$157 g$ at $4{ }^{\circ} \mathrm{C}$ for $10 \mathrm{~min}$ and the supernatants were transferred to new Eppendorf tubes

158 before drying at $45^{\circ} \mathrm{C}$ overnight and stored at $-40^{\circ} \mathrm{C}$. The dry cell extract samples

159 were resuspended in $210 \mu \mathrm{L}$ of potassium phosphate buffer $(\mathrm{pH}=7.4)$ containing

160 deuterium oxide $\left(\mathrm{D}_{2} \mathrm{O}\right)$ for magnetic field lock, 0.005\% 3-(trimethylsilyl)-[2,2,3,3- $\left.{ }^{2} \mathrm{H}_{4}\right]-$

161 propionic acid sodium salt (TSP) for the spectral calibration, $0.075 \mathrm{M} \mathrm{KH}_{2} \mathrm{PO}_{4}$, and 0.1

$162 \mathrm{mM}$ sodium azide $\left(\mathrm{NaN}_{3}\right)$. The resulting mixture was centrifuged at 20,817 $\mathrm{g}$ for $10 \mathrm{~min}$,

163 and $180 \mu \mathrm{L}$ supernatant was transferred to an NMR tube (Bruker Corporation,

164 Rheinstetten, Germany) with an outer diameter of $3 \mathrm{~mm}$ pending ${ }^{1} \mathrm{H}-\mathrm{NMR}$ spectral 165 acquisition.

166

167 The cell media were thawed and centrifuged at $18,000 \mathrm{~g}$ for $10 \mathrm{~min}$. A total of $540 \mu \mathrm{L}$

168 supernatant was mixed with $60 \mu \mathrm{L}$ potassium phosphate buffer containing $\mathrm{D}_{2} \mathrm{O}, 0.1 \%$

$169 \mathrm{TSP}, 1.5 \mathrm{M} \mathrm{KH}_{2} \mathrm{PO}_{4}$ and $2 \mathrm{mM} \mathrm{NaN}_{3}$. The mixture was transferred to an NMR tube with

170 an outer diameter of $5 \mathrm{~mm}$ pending ${ }^{1} \mathrm{H}$ NMR spectral acquisition.

172 The ${ }^{1} \mathrm{H}$ NMR spectra of cell extract and media samples were obtained using a Bruker

$173600 \mathrm{MHz}$ spectrometer (Bruker Corporation, Rheinstetten, Germany) at the operating

$174{ }^{1} \mathrm{H}$ frequency of $600.13 \mathrm{MHz}$ at a temperature of $300 \mathrm{~K}$. A standard NMR pulse 
sequence (recycle delay- $90^{\circ}-\mathrm{t}_{1}-90^{\circ}-\mathrm{t}_{\mathrm{m}}-90^{\circ}$ acquisition) was applied to acquire ${ }^{1} \mathrm{H} \mathrm{NMR}$

176 spectral data $\left(t_{1}=3 \mu \mathrm{s}, t_{m}=100 \mathrm{~ms}\right)$. The water peak suppression was achieved using

177 selective irradiation during a recycle delay of $4 \mathrm{~s}$ and $t_{\mathrm{m}}$. $A 90^{\circ}$ pulse was adjusted to $178 \sim 10 \mu \mathrm{s}$. A total of 32 scans were collected into $64 \mathrm{k}$ data points with a spectral width of 17920 ppm.

\section{PCR array}

182 RNeasy mini kit $\circledast$ and QIAshredder (QIAGEN, West Sussex, UK) were employed to 183 obtain total RNA from cells. A BioPhotometer (Eppendorf, Stevenage, UK) was used 184 to determine the quantity and quality of RNA. The A260/A280 and A260/A230 ratio 185 greater than 1.8 and 1.7 respectively, were regarded as sufficient quality for further 186 analysis. The $\mathrm{RT}^{2}$ First Strand Kit (QIAGEN) was utilized to convert total RNA to 187 complementary DNA (cDNA), which was then mixed with SYBR Green ROX FAST 188 Mastermix (QIAGEN). The mixture was added in an $\mathrm{RT}^{2}$ Profiler $^{\mathrm{TM}}$ PCR Array Human 189 Tumor Metastasis (QIAGEN), which was processed and analyzed with the Rotor-Gene 190 Q system (QIAGEN).

193 Paired oligonucleotide forward and reverse primers (Supplemental Table 2) for C-X-

194 C motif chemokine 12 (CXCL12), C-X-C chemokine receptor type 4 (CXCR4), and 195 GAPDH were designed using Primer Designer (Scientific and Educational Software, 
196 Durham, USA) against the sequence downloaded from GenBank and obtained from

197 Invitrogen. The process of RNA extraction and cDNA generation was the same as

198 those in the PCR array experiment. The cDNA sample was mixed with forward and

199 reverse primers and SYBR Green ROX qPCR Mastermix (QIAGEN). The PCR mixture

200 was processed and analyzed with the Rotor-Gene Q system (QIAGEN). All mRNA data

201 were expressed relative to the endogenous control gene, GAPDH.

202

203 Cell counting kit-8

204 The cultured A549 and H4 cells were added with cell counting kit-8 (CCK-8) solution

205 (Sigma-Aldrich, Dorset, UK). The blank well is with media and cell counting kit-8

206 solution but without cells. Cancer cells were incubated at $37{ }^{\circ} \mathrm{C}$ with $5 \% \mathrm{CO}_{2}$ for 2

207 hours. The optical density (OD) values were obtained via a microplate reader (BioTek,

208 Swindon, UK) at $450 \mathrm{~nm}$ wavelength. The cell viability value equals to (OD (Test) - OD

209 (Blank)) / (OD (Control) - OD (Blank)) X 100\%.

210

211 Wound healing scratch assay

212 Cancer cells were scratched in the center of the confluent monolayer and taken a

213 picture under microscope as the baseline. The cells were incubated in medium without

214 FBS for 24 hours, after which the picture was taken again at the same site. The

215 percentage of scratching gap closure was analyzed with ImageJ 2.0. 
218 Cells were collected after the dissociation by trypsin (Sigma-Aldrich). They were re-

219 suspended with serum-free media and seeded into the upper chamber of the transwell

220 assay kit (Sigma-Aldrich), which was pre-embedded with Matrigel (Sigma-Aldrich)

221 while the lower chamber was filled with serum-enrich media. After incubation for 24

222 hours, the upper chamber was fixed in $70 \%$ methanol for 30 minutes, which was

223 followed by 15 minutes staining with $0.1 \%$ crystal violet (Sigma-Aldrich). After removal

224 of the cells on the upper membrane, the cells remained on the bottom membrane were 225 identified as invasive cells.

\section{Statistical analysis}

228 The data of western blotting, CCK-8, Ki-67 staining, wound healing assay, and

229 transwell assay was analyzed by one-way analysis of variance (ANOVA) followed by

230 Dunnett test for comparison (GraphPad Prism 8.2.0, GraphPad Software, La Jolla,

231 California, USA). A two-sided $p$ value of less than 0.05 was considered to be a

232 statistical significance. The NMR data were imported and processed by MATLAB

233 R2018a (MathWorks, Cambridge, UK) programming language with MATLAB scripts

$234(21,22)$. After data was normalized and aligned, principal component analysis (PCA)

235 and orthogonal projections to latent structures discriminant analysis (OPLS-DA) were

236 used to analyze the processed spectral data. The RNA array data was uploaded to

237 GeneGlobe Data Analysis Centre (QIAGEN online program) and then analyzed. 


\section{Results}

240 Effects of propofol on cancer malignancy in lung cancer and neuroglioma cells

241 Propofol decreased the cell viability of A549 cells (Figure 1A, NC vs. P, $100.00 \pm 3.08$

242 vs. $92.23 \pm 1.83, \mathrm{p}<0.0001, \mathrm{n}=8$ ), while there was no significant change in $\mathrm{H} 4$ cells

243 (Figure 1B; NC vs. $\mathrm{P}, \mathrm{p}=0.13, \mathrm{n}=8$ ). A significant decrease of Ki-67 positive cell

244 numbers was observed in A549 cells treated with propofol compared with the naïve

245 control (Figure 1C and E, NC vs. P, $32.73 \pm 11.32$ vs. $5.992 \pm 1.019, p<0.01, n=5$ ).

246 In contrast, no change was detected in H4 cells (Figure 1D and F; $p=0.44$ for NC vs.

$247 \quad P, n=5)$

248

249 Propofol administration significantly decreased the migration of A549 cells (Figure 2A

250 and $\mathrm{B}, \mathrm{NC}$ vs. $\mathrm{P}, 53.84 \pm 3.59$ vs. $22.06 \pm 4.71, \mathrm{p}<0.001, \mathrm{n}=5$ ), but not $\mathrm{H} 4$ cells

251 (Figure 2C and $\mathbf{D} ; \mathrm{p}=1.00$ for $\mathrm{NC}$ vs. $\mathrm{P}, \mathrm{n}=5$ ). As shown by transwell assay, the 252 invasion of A549 was significantly decreased (NC vs. P, $1.00 \pm 0.14$ vs. $0.67 \pm 0.07, p$ $253<0.01, n=5$ ) by propofol (Figure $2 E$ and $\mathbf{F}$ ), while no significant difference (NC vs. P, $254 p=0.89, n=5$ ) in numbers of invasive cells following propofol administration was seen 255 in $\mathbf{H} 4$ cells (Figure $\mathbf{2} \mathbf{G}$ and $\mathbf{H}$ ). 
259 The immunofluorescent staining of A549 cells showed GLUT1 (a cell membrane

260 glucose transporter) and BRP44L (a mitochondrial pyruvate transporter) markers had

261 clear co-expressions in naïve control group and vehicle control group, while both

262 expressions in propofol group were significantly decreased (Figure $3 \mathbf{A}$ ). The protein

263 expressions of GLUT1 and BRP44L were significantly decreased in propofol group

264 compared with naïve control group; the expression levels of GLUT1 (NC vs. P, $1.00 \pm$

2650.69 vs. $0.29 \pm 0.13, \mathrm{p}<0.05, \mathrm{n}=6$ ) and BRP44L (NC vs. P, $1.00 \pm 0.16$ vs. $0.55 \pm$

$2660.09, p<0.01, n=6$ ) (Figure 3B-E). In H4 cells, GLUT1 and BRP44L expressions had

267 no changes among naïve control, vehicle control, and propofol groups in

268 immunofluorescent staining (Figure 4A, B) and western blotting analysis (Figure 4C-

269 F, NC vs. P, GLUT1, $p=0.74$, BRP44L, $p=0.99, n=6)$.

270

271 Propofol disturbed the metabolism of lung cancer cells but not neuroglioma

272 cells

273 The ${ }^{1} \mathrm{H}$ NMR spectroscopy was used to analyze metabolite changes in cell extract and

274 media samples of A549 and H4 cells. Pair-wise comparisons between control and

275 propofol group of A549 and H4 cells were carried out using OPLS-DA analysis with

276 one predictive component and one orthogonal component. $R^{2} X, Q^{2} X, Q^{2} Y$ and

277 permutation $p$ values of OPLS-DA models were summarized in Supplemental Table

278 3. The changes of metabolites observed in pair-wise comparisons were shown in

279 Supplemental Table 4. It showed a clear separation in A549 cell extracts between 
280 control and propofol groups (Figure 5A). The significant difference was contributed by

281 increased cellular concentrations of glutamate and glycine and decreased

282 concentrations of formate and acetate in propofol group (Figure 5B). The separation

283 in media samples between control and propofol groups of A549 cells was clearer than

284 the cell extracts (Figure 5C). With propofol treatment, the concentrations of lactate,

285 valine, isoleucine, leucine, glycerol and lipids were increased, while the concentrations

286 of pyruvate and isopropanol were decreased (Figure 5D). However, no significant

287 metabolic differences between the propofol and the control groups in either $\mathrm{H} 4$ cells or

288 media (permutation $p$ values $>0.05$ )

289

290 Propofol increased PEDF expressions in lung cancer cells but not in

291 neuroglioma cells

292 PEDF expression in A549 and H4 cells was evaluated using immunofluorescent

293 staining and western blotting. The immunofluorescent staining of A549 cells showed

294 that the expression level of PEDF in propofol group was higher than naïve control

295 group (Figure 6A). A significant increase in PEDF protein in the propofol group was

296 observed compared with naïve control group based on western blotting analysis (NC

297 vs. $\mathrm{P}, 1.00 \pm 0.42$ vs. $3.07 \pm 1.95, \mathrm{p}<0.05, \mathrm{n}=6$ ), but not between naïve and vehicle

298 control groups in A549 cells (Figure 6B and C). The immunofluorescent staining

299 (Figure 6D) and western blotting analysis (Figure 6E and F) of H4 cells showed no

300 significant change between any groups analysis (NC vs. $P, p=0.37, n=6$ ). 
302 Propofol suppressed both Akt and Erk pathways in lung cancer cells but not 303 neuroglioma cells

304 Akt and Erk pathways, which could be affected by PEDF, were evaluated. The 305 immunofluorescent staining showed both p-Akt (Figure 7A) and p-Erk (Figure 7B) of 306 lung cancer cells were suppressed by propofol treatment. Western blotting analysis 307 showed a significant decrease of $p-A k t / A k t$ between naïve control and propofol group 308 (NC vs. P, $1.00 \pm 0.45$ vs. $0.35 \pm 0.07, p<0.01, n=6$ ) (Figure 7C and 7D). Moreover, 309 the evaluation for p-Erk/Erk showed the similar pattern that a significant change was 310 identified between naïve control and propofol group (NC vs. P, $1.00 \pm 0.52$ vs. $0.38 \pm$

$3110.07, p<0.01, n=6$ ), but not between naïve and vehicle control group (Figure 7E and 312 F). In $\mathrm{H} 4$ cells, there was no significant change between any groups of $p$-Akt/Akt (NC 313 vs. $\mathrm{P}, \mathrm{p}=1.00, \mathrm{n}=6$, Figure $7 \mathrm{G}$ and $7 \mathrm{H}$ ) and $\mathrm{p}$-Erk/Erk (NC vs. $\mathrm{P}, \mathrm{p}=0.72, \mathrm{n}=6$, 314 Figure $7 \mathrm{I}$ and $\mathbf{7 J}$ ).

316 HIF-1 $1 \alpha$ expression was downregulated by propofol in lung cancer cells but not

317 neuroglioma cells

318 HIF-1 $1 \alpha$ is one of the downstream pathways of Akt and Erk pathways. The 319 immunofluorescent staining showed HIF-1 $1 \alpha$ expression in A549 cells was decreased 320 after propofol treatment (Figure 8A). HIF-1a expression in A549 cells measured by 321 western blotting was also decreased in the propofol group (NC vs. P, $1.00 \pm 0.20$ vs. 
$3220.67 \pm 0.14, p<0.01, n=6$ ) compared with naïve control group (Figure 8B and $\mathbf{C}$ ).

323 However, HIF-1 1 expression in $\mathrm{H} 4$ cells was not changed following propofol exposure

324 (Figure 8D, E and F; $p=0.91$ for NC vs. P of western blotting analysis, $n=6$ ).

Effects of propofol on tumor metastatic related gene expressions of lung cancer and neuroglioma cells

Out of 84 tumor metastasis-related genes, 6 pro-tumor genes, namely, VEGFA, CTBP1, CST7, CTSK, CXCL12, and CXCR4, were downregulated in the propofol group compared with control. In addition, 8 anti-tumor genes including NR4A3, RB1, NME1, MTSS1, NME4, SYK, APC, and FAT1 were upregulated (Figure 9A) in lung cancer cells. Among these altered gene expressions, CXCL12 and CXCR4 were significantly

333 downregulated by propofol administration (Figure 9B, CXCL12, C vs. P, $1.000 \pm 0.004$

334 vs. $0.986 \pm 0.004, \mathrm{p}<0.05, \mathrm{n}=3$; Figure $9 \mathrm{C}, \mathrm{CXCR} 4, \mathrm{C}$ vs. $\mathrm{P}, 1.000 \pm 0.004$ vs. 0.986

$335 \pm 0.004, p<0.05, n=3)$. These expression were subsequently validated by qRT-PCR, 336 which showed consistent results with PCR array (Figure 9D, CXCL12, C vs. P, $1.00 \pm$ 3370.36 vs. $0.08 \pm 0.04, \mathrm{p}<0.05, \mathrm{n}=3$; Figure $9 \mathrm{E}, \mathrm{CXCR4}, \mathrm{C}$ vs. $\mathrm{P}, 1.00 \pm 0.41$ vs. 0.23 $338 \pm 0.16, p<0.05, n=3$ ). However, there were no significant changes in CXCL12 (Figure 339 9F, $p=0.35, n=5$ ) and CXCR4 (Figure 9G, $p=0.79, n=5$ ) expressions in $\mathrm{H} 4$ cells 340 between control and propofol groups. 


\section{Discussion}

344 In the current study, we found that propofol, one of the most commonly used

345 intravenous general anesthetic, downregulated GLUT1, BRP44L, HIF-1a, p-Akt and

346 p-Erk1/2 expressions and upregulated PEDF in lung cancer cells but not in brain

347 cancer cells (Figure 10). Furthermore, 6 pro-tumor genes were downregulated and 8

348 anti-tumor genes were upregulated after propofol administration, while the cell

349 metabolism of lung cancer cells was altered by propofol. Ultimately, the cell viability,

350 proliferation, migration and invasion of lung cancer cells were suppressed by propofol.

351 However, these effects of propofol were not found in neuroglioma cells. Our data

352 indicated that the malignancy of lung cancer cells was attenuated by propofol, which

353 might be associate with the alterations of cell metabolisms and cell signaling pathways.

354

355 In this study, propofol was found to inhibit GLUT1 and BRP44L expressions, which

356 were located at the cellular and mitochondrial membrane, respectively. In line with our

357 data, propofol was previously reported to suppress GLUT1 expression in human

358 myeloid leukemia cells (23). BRP44L, also known as mitochondrial pyruvate carrier 1 ,

359 is responsible for transferring pyruvate into mitochondria for the tricarboxylic acid (TCA)

360 cycle, which is a major energy synthesis pathway for normal cells (24). However, in

361 contrast to normal cells, cancer cells are more likely to shift from mitochondrial

362 oxidative phosphorylation to aerobic glycolysis, such phenomenon is called "Warburg

363 effect" (25). "Warburg effect" requires the downregulation of BRP44L in cancer cells 
364 and the BRP44L levels are relatively low in several cancer types, including lung cancer

$365(26,27)$. Although cancer cells do not mainly rely on the TCA cycle to generate ATP, it

366 still requires the TCA cycle to produce the intermediates for the synthesis of nucleic

367 acids, fatty acids, and carbon skeleton (28). Interestingly, resveratrol is a phenolic

368 structured natural healthy supplement that not only blocks the activity of GLUT1, but

369 also downregulates the expression level of GLUT1. It was found that the exposure of

370 resveratrol to leukemic and ovarian cancer cells inhibits the uptake of glucose (29).

371 Resveratrol was found to inhibit the proliferation, metastasis and epigenetic alterations,

372 and induce the apoptosis in vitro and in vivo studies of breast cancer (30). Lonidamine,

373 an anti-tumor drug, was found to kill cancer cells by inhibiting the activity of BRP44L.

374 This was in agreement with our ${ }^{1} \mathrm{H}$ NMR spectral data, which showed that lactate in

375 the media of lung cancer cells was increased, while pyruvate was decreased by

376 propofol, indicating that pyruvate was more converted to lactate and less entered the

377 TCA cycle. This was very likely due to the decrease of BRP44L demonstrated in our

378 study while propofol itself induced mitochondrial bilayer perturbations might contribute

379 to this. In cancer cells, pyruvate is converted to formate and acetyl-CoA catalyzed by

380 pyruvate formate lyase, and acetyl-CoA can be metabolized to acetate or enter TCA

381 cycle (31). The concentrations of formate and acetate were decreased following the

382 administration of propofol, indicating pyruvate metabolism towards the production of

383 acetyl-CoA was reduced. In cancer cells, apart from glucose, glutamine is another

384 nutrition source, which is converted to glutamate and used in TCA cycle, namely 

increased in lung cancer cell extracts after treated with propofol, which was an

387 evidence of lower activity of glutaminolysis and TCA cycle. The metabolism of other 388 amino acids was also affected, including glycine, valine, isoleucine, and leucine. 389 Glycine was elevated in lung cancers administered with propofol. As glycine can be 390 converted to pyruvate, which is relevant with glucose metabolism or TCA cycle (33). 391 Isoleucine, leucine and valine was elevated in the media of propofol group, which 392 further indicted that propofol inhibited the metabolism of lung cancer cells, leading to 393 less utilization of amino acids. Isopropanol, a potential primary lung cancer biomarker 394 (34), was decreased in lung cancer cells with propofol treatment, suggesting that the 395 progression of lung cancer cells was inhibited by the treatment. Although the 396 metabolism of isopropanol was not fully understood, some reports claimed that 397 isopropanol might be transformed from acetone, which was converted from pyruvate $398(35,36)$. Another source of acetone is from beta-oxidation of fatty acids in mitochondria 399 (37). Propofol might injure mitochondrial beta-oxidation, which generated less acetone 400 to convert to isopropanol. These findings collectively indicated that propofol likely 401 causes mitochondrial injury and disturbs cancer cell metabolisms. It is true that 402 propofol is different from the other anesthetics as it has a unique phenolic structure, 403 which gives it a lipophilic property that can be solubilized inside the lipid membrane 404 bilayer and induce the lipid perturbations $(38,39)$, whilst the lipid bilayer is the platform 405 for protein-protein interaction and cellular signaling modulation, and its perturbations 
can affect several signaling pathways and biochemical reactions (40).

408 It was reported that under high glucose concentration, PEDF expression was

409 decreased in retinal Muller cells $(41,42)$. In line with this, GLUT1 was downregulated

410 in lung cancer cells by propofol and less glucose uptake resulted in a relatively lower

411 concentration of glucose in cellular plasma of lung cancer cells. However, we did not

412 identify elevated glucose in media, which might due to other glucose transporters

413 (there is a total of 14 GLUTs expressed in human cells (43)) compensate the

414 decreased GLUT1 function. The compensation of GLUTs might cause a transient

415 change of glucose concentration in lung cancer cells that might induce the secretion

416 of PEDF, which was confirmed with our both immunofluorescent staining and western

417 blot data (Figure 6). PEDF has an anti-tumor and anti-angiogenesis property and

418 some tumor malignancy-related cellular signaling pathways in lung cancer cells may

419 likely be interrupted by the increased level of PEDF. Indeed, Akt and Erk in lung cancer

420 cells were inhibited by propofol as shown in our study and also reported previously (44,

421 45). Our group previously demonstrated that volatile anesthetic isoflurane enhanced

422 the malignancy of renal cancer cells by activating HIF-1 $\alpha$ via Akt signaling pathway

423 (46). Overexpressed HIF-1 $\alpha$ had been found in many aggressive cancer types and

424 was found to correlate with tumor progression $(47,48)$. In contrast to inhaled

425 anesthetics, propofol inactivated HIF-1 $\alpha$ in lung cancer cells in the current study; this

426 is consistent with prostate cancer cells in which propofol inhibited the synthesis of HIF- 
427 1a through Akt pathway which was initially induced by isoflurane and then suppressed

428 by the superposition of propofol as we reported (11). HIF-1 $\alpha$ is a key transcriptional

429 regulator which are involved in cell survival, proliferation, migration and invasion (49).

430 Our PCR array results showed that several pro-tumor (for example, VEGFA, CXCL12,

431 and $C X C R 4$ ) genes were downregulated and anti-tumor genes (for example, RB1,

432 APC, and FAT1) were upregulated (Supplemental Table 5). These were in contrast to

433 volatile anesthetics which induced the tumor metastatic related genes that were

434 associated with the enhanced malignancy of ovarian cancer cells (10). Interestingly, a

435 retrospective clinical study showed that patients received tumor resection were

436 grouped into total intravenous anesthesia (propofol and remifentanil) or inhalational

437 anesthesia (isoflurane or sevoflurane) groups. It was found that patients received

438 inhalational anesthesia during cancer surgery had a lower 3 year-survival rate than

439 those received propofol-based intravenous anesthesia (9). In another study, it was

440 concluded that propofol-based intravenous anesthesia for colon cancer surgery was

441 associated with better survival rate than desflurane-based inhalational anesthesia (50).

442 Arguably, these clinical data are well supported by our current findings that propofol

443 inhibits the malignancy of cancer cells, albeit derived from lung cancer.

445 In contrast, the expression of GLUT1, BRP44L, PEDF, p-Akt, p-Erk, and HIF-1a was

446 not changed in neuroglioma cells by propofol. In addition, no significant changes were

447 identified in our metabolism and PCR data. Unlike lung cancer cells, the cell viability, 
448 proliferation, migration, and invasion of neuroglioma cells were not significantly

449 inhibited by propofol either. Why neuroglioma cells behaved so different to propofol

450 compared to lung cancer cells is unknown and warrants further study. One can argue

451 that, in general, brain cancer is very insensitive to chemotherapy and/or radiotherapy,

452 indicating that this type of cancer is more robust than other cancer types. Nevertheless,

453 our study may support clinical retrospective observations, in which patients were under

454 either propofol or sevoflurane maintenance of anesthesia for glioma resection and their

455 progression-free or overall survival were not different between two anesthetic

456 regimens $(51,52)$. The current study was based on in vitro assays and the future

457 investigation need to be carried out in vivo to evaluate the effect of propofol at the

458 systemic level. Clinically, there are many other risk factors, for example, surgery

459 induced inflammation and abnormal immune function during perioperative period, that

460 also affect the prognosis of cancer patients after surgery. It was reported that propofol

461 increased the cytotoxicity effects of natural killer (NK) cells, which might also benefit

462 the outcome of cancer patients (53). All these point to that propofol may be a good

463 choice of anesthetics used during surgery for certain cancer type but in order to better

464 simulate clinical scenarios, in vivo and clinical studies are required.

465

466 Conclusions

467 Our data suggested that unlike brain cancer cells, propofol disturbed the metabolism, 468 decreased GLUT1 and BRP44L expressions, and increased PEDF expression of lung 
cancer cells. PEDF then inhibited HIF-1 $\alpha$ via both Akt and Erk signaling and

470 consequently upregulated anti-tumor genes and downregulated pro-tumor genes. The

471 alteration of tumor metastatic related genes together with the disturbance of cellular

472 metabolism may ultimately lead to the inhibition of malignancy of lung cancer cells.

473 Our study could lead to new anesthetic regimens for cancer lung surgery. However, in

474 vitro experimental setting cannot represent clinical scenario and, therefore, further 475 clinical study/trial are required to valid the benefit effects of propofol found in our study.

\section{List of Abbreviations}

478 GLUT1: glucose transporter 1; BRP44L: brain protein 44-like; PEDF: pigment 479 epithelium-derived factor; p-Akt: phospho-Akt; p-Erk: phospho-Erk; HIF-1a: hypoxia480 inducible factor 1 alpha; ${ }^{1} \mathrm{H}$ NMR: proton nuclear magnetic resonance; CCK-8: cell 481 counting kit-8; MPC1: mitochondrial pyruvate carrier 1; FBS: fetal bovine serum; 4', 6482 diamidino-2-phenylindole: DAPI; ECL: enhanced chemiluminescence; $\mathrm{D}_{2} \mathrm{O}$ : deuterium 483 oxide; TSP: 3-(trimethylsilyl)-[2,2,3,3-2 $\left.\mathrm{H}_{4}\right]$-propionic acid sodium salt; $\mathrm{NaN}_{3}$ : sodium 484 azide; cDNA: complementary DNA; CXCL12: C-X-C motif chemokine 12; CXCR4: C485 X-C chemokine receptor type 4; OD: optical density; SD: standard deviation; ANOVA: 486 one-way analysis of variance; PCA: principal component analysis; OPLS-DA: 487 orthogonal projections to latent structures discriminant analysis; TCA: tricarboxylic acid; $488 \quad$ NK cell: natural killer cell. 


\section{Declarations}

491 Ethics approval and consent to participate

492 Not applicable.

\section{Consent for publication}

495 Not applicable.

497 Availability of data and materials

498 The datasets used and/or analyzed during the current study are available from the 499 corresponding author on reasonable request.

500

501 Completing interests

502 The authors declare that they have no completing interests.

503

504 Funding

505 CH was supported by a PhD scholarship (201700260043, 2017-2020) from China

506 Scholarship Council, Beijing, China. JVL was funded by MRC New Investigator Grant 507 (MR/P002536/1), MRC, London, UK and ERC Starting Grant (715662). DM was

508 funded by BOC Chair grant, Royal College of Anaesthetists, London, UK. Partial 509 research consumable cost was from the Second Affiliated Hospital and Yuying

510 Children's Hospital of Wenzhou Medical University, Wenzhou, Zhejiang, China. 


\section{Author's contributions}

513 DM, JVL, QL and CH designed the study. CH conducted cell culture, CCK-8, wound

514 healing assay, Transwell assay, immunofluorescent staining, NMR sample extraction

515 and preparation. $\mathrm{CH}$ and $\mathrm{XL}$ conducted double-stain immunofluorescence. $\mathrm{CH}$ and BW

516 conducted Western blot. $\mathrm{CH}$ and ZL conducted NMR spectroscopic analysis. $\mathrm{CH}$ and

517 MI conducted PCR array and qRT-PCR. CH and JVL conducted NMR data analysis.

$518 \mathrm{CH}$ conducted other data analysis alone. $\mathrm{CH}$ drafted the manuscript. $\mathrm{CH}, \mathrm{MI}, \mathrm{ZL}, \mathrm{BW}$,

$519 \mathrm{XL}, \mathrm{HL}, \mathrm{JL}, \mathrm{QL}, \mathrm{JVL}$ and DM participated in the review of the manuscript.

\section{Acknowledgements}

522 Figure 10 was created by using Servier Medical Art materials, which are licensed under

523 a Creative Commons Attribution 3.0 Unported License.

\section{References}

526 1. Collaboration GBoDC. Global, Regional, and National Cancer Incidence, 527 Mortality, Years of Life Lost, Years Lived With Disability, and Disability-Adjusted 528 Life-years for 32 Cancer Groups, 1990 to 2015: A Systematic Analysis for the 529 Global Burden of Disease Study. JAMA oncology. 2017;3(4):524-48.

530 2. Torre LA, Bray F, Siegel RL, Ferlay J, Lortet-Tieulent J, Jemal A. Global Cancer 531 Statistics, 2012. Ca-a Cancer Journal for Clinicians. 2015;65(2):87-108.

532 3. Siegel RL, Miller KD, Jemal A. Cancer Statistics, 2017. Ca-a Cancer Journal for 533 Clinicians. 2017;67(1):7-30.

534 4. A new clinical guideline from the Royal College of Paediatrics and Child Health 535 with a national awareness campaign accelerates brain tumor diagnosis in UK 536 children--"HeadSmart: Be Brain Tumour Aware". Neuro-oncology. 537 2016;18(3):445-54. 
5. Aliperti LA, Predina JD, Vachani A, Singhal S. Local and systemic recurrence is the Achilles heel of cancer surgery. Annals of surgical oncology. 2011;18(3):603-7. 6. Ng JCH, See AAQ Ang TY, Tan LYR, Ang BT, King NKK. Effects of surgery on neurocognitive function in patients with glioma: a meta-analysis of immediate post-operative and long-term follow-up neurocognitive outcomes. Journal of neuro-oncology. 2019;141(1):167-82.

7. Lee JS, Kim SI, Park HS, Lee JS, Park S, Park BW. The impact of local and regional recurrence on distant metastasis and survival in patients treated with breast conservation therapy. Journal of breast cancer. 2011;14(3):191-7.

8. Horowitz M, Neeman E, Sharon E, Ben-Eliyahu S. Exploiting the critical perioperative period to improve long-term cancer outcomes. Nature reviews Clinical oncology. 2015;12(4):213-26.

9. Wigmore TJ, Mohammed K, Jhanji S. Long-term Survival for Patients Undergoing Volatile versus IV Anesthesia for Cancer Surgery: A Retrospective Analysis. Anesthesiology. 2016;124(1):69-79.

10. Iwasaki M, Zhao H, Jaffer T, Unwith S, Benzonana L, Lian $Q$, et al. Volatile anaesthetics enhance the metastasis related cellular signalling including CXCR2 of ovarian cancer cells. Oncotarget. 2016;7(18):26042-56.

11. Huang H, Benzonana LL, Zhao H, Watts HR, Perry NJ, Bevan C, et al. Prostate cancer cell malignancy via modulation of HIF-1alpha pathway with isoflurane and propofol alone and in combination. British journal of cancer. 2014;111(7):133849.

12. Soni S, Padwad YS. HIF-1 in cancer therapy: two decade long story of a transcription factor. Acta oncologica (Stockholm, Sweden). 2017;56(4):503-15.

13. Jing Y, Liu LZ, Jiang Y, Zhu Y, Guo NL, Barnett J, et al. Cadmium increases HIF-1 and VEGF expression through ROS, ERK, and AKT signaling pathways and induces malignant transformation of human bronchial epithelial cells. Toxicological sciences : an official journal of the Society of Toxicology. 2012;125(1):10-9.

14. Wan J, Wu W. Hyperthermia induced HIF-1a expression of lung cancer through AKT and ERK signaling pathways. Journal of experimental \& clinical cancer research : CR. 2016;35(1):119.

15. Mayer IA, Arteaga CL. The PI3K/AKT Pathway as a Target for Cancer Treatment. Annual review of medicine. 2016;67:11-28.

16. Samatar AA, Poulikakos PI. Targeting RAS-ERK signalling in cancer: promises and challenges. Nature reviews Drug discovery. 2014;13(12):928-42.

17. Yuan Y, Liu X, Miao H, Huang B, Liu Z, Chen J, et al. PEDF increases GLUT4mediated glucose uptake in rat ischemic myocardium via PI3K/AKT pathway in a PEDFR-dependent manner. International journal of cardiology. 2019;283:136-43. 18. Sanchez A, Tripathy D, Yin X, Luo J, Martinez J, Grammas P. Pigment epithelium-derived factor (PEDF) protects cortical neurons in vitro from oxidant injury by activation of extracellular signal-regulated kinase (ERK) $1 / 2$ and induction of Bcl-2. Neuroscience research. 2012;72(1):1-8. 
19. Conte MI, Cabrillana ME, Saez Lancellotti TE, Simon L, Funes AK, CayadoGutierrez N, et al. Pigment epithelium derived factor (PEDF) expression in the male tract of Wistar rats. Biochem Biophys Res Commun. 2018;504(1):257-62. 20. Wei Y, Elahy M, Friedhuber AM, Wong JY, Hughes JD, Doschak MR, et al. Triplethreat activity of PEDF in bone tumors: Tumor inhibition, tissue preservation and cardioprotection against doxorubicin. Bone. 2019;124:103-17.

21. Trygg J, Holmes E, Lundstedt T. Chemometrics in metabonomics. J Proteome Res. 2007;6(2):469-79.

22. Haggart GA. csmsoftware/IMPaCTS: Version 1.1.1 (Version v1.1.1) Zenodo2019 [Available from: http://doi.org/10.5281/zenodo.3077413.

\section{Tanaka T, Takabuchi S, Nishi K, Oda S, Wakamatsu T, Daijo H, et al. The} intravenous anesthetic propofol inhibits lipopolysaccharide-induced hypoxiainducible factor 1 activation and suppresses the glucose metabolism in macrophages. Journal of anesthesia. 2010;24(1):54-60.

\section{Bricker DK, Taylor EB, Schell JC, Orsak T, Boutron A, Chen Y-C, et al. A} mitochondrial pyruvate carrier required for pyruvate uptake in yeast, Drosophila, and humans. Science. 2012;337(6090):96-100.

25. Vander Heiden MG, Cantley LC, Thompson CB. Understanding the Warburg effect: the metabolic requirements of cell proliferation. science. 2009;324(5930):1029-33.

26. Lu Y, Yi Y, Liu P, Wen W, James M, Wang D, et al. Common human cancer genes discovered by integrated gene-expression analysis. PloS one. 2007;2(11):e1149-e. 27. Zou H, Chen Q, Zhang A, Wang S, Wu H, Yuan Y, et al. MPC1 deficiency accelerates lung adenocarcinoma progression through the STAT3 pathway. Cell death \& disease. 2019;10(3):148-.

28. Anderson NM, Mucka P, Kern JG, Feng H. The emerging role and targetability of the TCA cycle in cancer metabolism. Protein Cell. 2018;9(2):216-37.

29. Zambrano A, Molt M, Uribe E, Salas M. Glut 1 in Cancer Cells and the Inhibitory Action of Resveratrol as A Potential Therapeutic Strategy. Int J Mol Sci. 2019;20(13).

30. Sinha D, Sarkar N, Biswas J, Bishayee A. Resveratrol for breast cancer prevention and therapy: Preclinical evidence and molecular mechanisms. Seminars in cancer biology. 2016;40-41:209-32.

31. Tack I, Nimmegeers P, Akkermans S, Logist F, Van Impe JFM. A low-complexity metabolic network model for the respiratory and fermentative metabolism of Escherichia coli. PloS one. 2018;13(8):e0202565.

32.Dang CV. Glutaminolysis: supplying carbon or nitrogen or both for cancer cells? Cell cycle (Georgetown, Tex). 2010;9(19):3884-6.

33. Cheng ZX, Guo C, Chen ZG, Yang TC, Zhang JY, Wang J, et al. Glycine, serine and threonine metabolism confounds efficacy of complement-mediated killing. Nature communications. 2019;10(1):3325. 
GA, et al. Lung cancer detection by proton transfer reaction mass-spectrometric analysis of human breath gas. International Journal of Mass Spectrometry. 2007;265(1):49-59.

35. Lewis GD, Laufman AK, McAnalley BH, Garriott JC. Metabolism of acetone to isopropyl alcohol in rats and humans. J Forensic Sci. 1984;29(2):541-9.

36. Li WW, Liu Y, Liu Y, Cheng SQ, Duan YX. Exhaled isopropanol: new potential biomarker in diabetic breathomics and its metabolic correlations with acetone. Rsc Advances. 2017;7(28):17480-8.

37. Laffel L. Ketone bodies: a review of physiology, pathophysiology and application of monitoring to diabetes. Diabetes Metab Res Rev. 1999;15(6):41226.

38. Reitz M, Velizarov S, Glück B, Berg H, Brambrink AM. Effects of propofol (intravenous propofol emulsion) on cell membrane measures by electrofusion and electroporation. Arzneimittel-Forschung/Drug Research. 1999;49(3):281-5. 39. Bahri MA, Seret A, Hans P, Piette J, Deby-Dupont G, Hoebeke M. Does propofol alter membrane fluidity at clinically relevant concentrations? An ESR spin label study. Biophysical Chemistry. 2007;129(1):82-91.

40. Hicks DA, Nalivaeva NN, Turner AJ. Lipid rafts and Alzheimer's disease: protein-lipid interactions and perturbation of signaling. Frontiers in physiology. 2012;3:189.

41. Mu H, Zhang X-M, Liu J-J, Dong L, Feng Z-L. Effect of high glucose concentration on VEGF and PEDF expression in cultured retinal Müller cells. Molecular biology reports. 2009;36(8):2147.

42. Yamagishi S-i, Matsui T. Pigment epithelium-derived factor (PEDF) and cardiometabolic disorders. Current pharmaceutical design. 2014;20(14):2377-86. 43. Thorens B, Mueckler M. Glucose transporters in the 21st Century. American journal of physiology Endocrinology and metabolism. 2010;298(2):E141-5. 44. Hsing C-H, Lin M-C, Choi P-C, Huang W-C, Kai J-I, Tsai C-C, et al. Anesthetic propofol reduces endotoxic inflammation by inhibiting reactive oxygen speciesregulated Akt/IKK $\beta / N F-\kappa B$ signaling. PloS one. 2011;6(3):e17598.

45. Fibuch EE, Wang JQ. Inhibition of the MAPK/ERK cascade: a potential transcription-dependent mechanism for the amnesic effect of anesthetic propofol. Neuroscience bulletin. 2007;23(2):119-24. 46. Benzonana LL, Perry NJ, Watts HR, Yang B, Perry IA, Coombes C, et al. Isoflurane, a commonly used volatile anesthetic, enhances renal cancer growth and malignant potential via the hypoxia-inducible factor cellular signaling pathway in vitro. Anesthesiology. 2013;119(3):593-605.

47. Unwith S, Zhao H, Hennah L, Ma D. The potential role of HIF on tumour progression and dissemination. International journal of cancer Journal international du cancer. 2015;136(11):2491-503.

48. Zhao H, Iwasaki M, Yang J, Savage S, Ma D. Hypoxia-inducible factor-1: a possible link between inhalational anesthetics and tumor progression? Acta 
664 anaesthesiologica Taiwanica : official journal of the Taiwan Society of 665 Anesthesiologists. 2014;52(2):70-6.

666 49. Semenza GL. Targeting HIF-1 for cancer therapy. Nature reviews Cancer. 667 2003;3(10):721-32.

668 50. Wu ZF, Lee MS, Wong CS, Lu CH, Huang YS, Lin KT, et al. Propofol-based Total 669 Intravenous Anesthesia Is Associated with Better Survival Than Desflurane 670 Anesthesia in Colon Cancer Surgery. Anesthesiology. 2018;129(5):932-41.

671 51. Dong J, Zeng M, Ji N, Hao S, Zhou Y, Gao Z, et al. Impact of Anesthesia on Long672 term Outcomes in Patients With Supratentorial High-grade Glioma Undergoing 673 Tumor Resection: A Retrospective Cohort Study. Journal of neurosurgical 674 anesthesiology. 2020;32(3):227-33.

675 52. Saito J, Masters J, Hirota K, Ma D. Anesthesia and brain tumor surgery: 676 technical considerations based on current research evidence. Current opinion in 677 anaesthesiology. 2019;32(5):553-62.

678 53. Li R, Liu H, Dilger JP, Lin J. Effect of Propofol on breast Cancer cell, the immune 679 system, and patient outcome. BMC anesthesiology. 2018;18(1):77.

680

681

682

683

684

685

686

687

688

689

690

691

692 


\section{Figure legends}

694 Figure 1 . The cell viability and proliferative capability of lung cancer and 695 neuroglioma cells after propofol exposure.

696 Lung cancer A549 and neuroglioma H4 cells were treated with intralipid (vehicle 697 control), $4 \mu \mathrm{g} / \mathrm{mL}$ propofol, or pure culture media (naïve control). Cell viability of lung 698 cancer (A) and neuroglioma cells (B) was evaluated with the CCK-8 assay. Cell 699 proliferative capability of A549 (C) and H4 cells (D) with Ki-67 immunofluorescent 700 staining. The comparison of Ki-67 positive cell percentage in A549 cells (E) and H4 701 cells $(\mathbf{F})$. Data were expressed as mean \pm standard deviation and dot plot $(n=5-8)$. ${ }^{* *} \mathrm{p}<0.01,{ }^{* * *} \mathrm{p}<0.0001$ versus naïve control. Scale bar: $100 \mu \mathrm{m}$. NC, naïve control;

703 VC, vehicle control; P, propofol.

Figure 2. The migrate and invasive ability of lung cancer and neuroglioma cells

706 after propofol administration.

707 Lung cancer A549 and neuroglioma H4 cells were administered with intralipid (vehicle 708 control), $4 \mu \mathrm{g} / \mathrm{mL}$ propofol, or no treatment (naïve control). The migration of lung cancer 709 (A) and neuroglioma cells (C) was assessed via wound healing assay with statistical 710 analysis of the percentage of gap closure of A549 (B) and $\mathrm{H} 4$ cells (D). The invasion

711 of $\mathrm{A} 549$ (E) and H4 cells (G) was evaluated by Transwell assay with the statistical 712 analysis of the relative ratio of invasive cell number to NC in A549 (F) and H4 cells $(\mathbf{H})$.

713 Data were presented as mean \pm standard deviation and dot plot $(n=5)$. ${ }^{* *} p<0.01$, 
$714{ }^{* * *} p<0.001$ versus naïve control. Scale bar: $100 \mu \mathrm{m}$. NC: naïve control; V: vehicle 715 control; P: propofol.

717 Figure 3. Propofol down-regulated GLUT1 and BRP44L in lung cancer cells.

718 Lung cancer A549 cells were administered with $4 \mu \mathrm{g} / \mathrm{mL}$ propofol or intralipid (vehicle

719 control) or media without drugs (naïve control) for 2 hours followed by 24 hours 720 recovery time. GLUT1 (green) and BRP44L (red) expressions were identified by dual721 immunofluorescent staining (A). The expression levels of GLUT1 (B and C) and 722 BRP44L (D and E) were validated with western blotting analysis. The intensity of 723 western blotting bands was normalized by housekeeping gene GAPDH. Data were 724 analyzed with one-way analysis of variance (ANOVA) followed by Dunnett multi725 comparison test. Data were presented as mean \pm standard deviation and dot plot $(\mathrm{n}=$ 726 6). ${ }^{*} p<0.05 ;{ }^{* *} p<0.01$ versus naïve control. Scale bar: $50 \mu \mathrm{m}$. NC: naïve control; VC: 727 vehicle control; P: propofol; GLUT1: glucose transporter 1; BRP44L: brain protein 44728 like.

730 Figure 4. GLUT1 and BRP44L expressions in neuroglioma cells after 731 administered with propofol.

732 Neuroglioma $\mathrm{H} 4$ cells were administered with a clinically relevant concentration of 4 $733 \mu \mathrm{g} / \mathrm{mL}$ propofol. GLUT1 (green) and BRP44L (red) expressions were analyzed by 734 immunofluorescent staining (A and $\mathbf{B}$ ) and validated with western blotting (C-F). The 
735 intensity of Western blot bands was normalized by housekeeping gene GAPDH. Data

736 were analyzed with one-way analysis of variance (ANOVA) followed by Dunnett multi-

737 comparison test. Data were presented as mean \pm standard deviation and dot plot $(\mathrm{n}=$

738 6). Scale bar: $50 \mu \mathrm{m}$. NC: naïve control; VC: vehicle control; P: propofol; GLUT1:

739 glucose transporter 1; BRP44L: brain protein 44-like.

740

741 Figure 5. The metabolism alterations of lung cancer cells after propofol

742 administration.

743 Orthogonal projection to latent structures-discriminant analysis (OPLS-DA) loadings

744 plots from ${ }^{1} \mathrm{H}$ NMR spectral data of the A549 cell extract (A and B) and A549 media

745 samples (C and $\mathbf{D})$ for comparisons as control vs. propofol $(n=9-10)$. A and C: OPLS-

746 DA scores plot; B and D: OPLS-DA loadings plot. The color bar indicates the

747 correlation coefficient values $\left(r^{2}\right)$ to be high in red and low in blue. C: control; P:

748 propofol; F: formate; Glu: glutamate; Gly: glycine; Ace: acetate; Lac: lactate; Gro:

749 glycerol; Pyr: pyruvate; FA: fatty acids; IPA: isopropanol; Val: valine; Ile: isoleucine;

750 Leu: leucine.

751

752 Figure 6. Propofol up-regulated PEDF in lung cancer cells but not neuroglioma

753 cells.

754 Lung cancer A549 and neuroglioma H4 cells were administered with a clinically

755 relevant concentration $4 \mu \mathrm{g} / \mathrm{mL}$ of propofol for 2 hours. PEDF expression was analyzed 
756 by immunofluorescent staining (A. A549 cells and D. H4 cells) and western blotting

757 analysis (B. A549 cells and E. H4 cells). PEDF (green) was overlaid with DAPI (blue).

758 The intensity of western blotting bands was normalized by housekeeping gene GAPDH

759 (C. A549 cells and F. A549 cells). Data were analyzed with one-way analysis of

760 variance (ANOVA) followed by Dunnett multi-comparison test. Data were presented as

761 mean \pm standard deviation and dot plot $(n=6) .{ }^{*} p<0.05$ versus naïve control. Scale

762 bar: $50 \mu \mathrm{m}$. NC, naïve control; VC, vehicle control; P, propofol; PEDF; pigment 763 epithelium-derived factor.

764

765 Figure 7. Propofol down-regulated Akt and Erk expression in lung cancer cells

766 but not neuroglioma cells.

767 Lung cancer A549 and neuroglioma $\mathrm{H} 4$ cells were treated with $4 \mu \mathrm{g} / \mathrm{mL}$ propofol or

768 vehicle control or naïve control. A. p-Akt (green) of lung cancer cells as cytoplasmic

769 dyeing overlaid with DAPI (blue) staining in nucleus. B. p-Erk1/2 (green) was stained

770 in cytoplasm overlaid with DAPI (blue) in the nucleus. C. The representative western

771 blotting bands of p-Akt and Akt expression of A549 cells. D. The relative ratio of p-Akt

772 to Akt compared to naïve control of A549 cells. E. The representative bands of $p$-Erk $1 / 2$

773 and Erk1/2 expression of A549 cells. F. The representative western blotting bands of

774 p- Erk1/2 and Erk1/2 expression of A549 cells. G. The representative bands of p-Akt

775 and Akt expression of $\mathrm{H} 4$ cells. $\mathbf{H}$. The relative ratio of $\mathrm{p}$-Akt to Akt compared to naïve

776 control of $\mathrm{H} 4$ cells. I. The representative bands of $\mathrm{p}$-Erk1/2 and Erk1/2 expression of 
$777 \mathrm{H} 4$ cells. J. The representative western blotting bands of $\mathrm{p}-\mathrm{Erk} 1 / 2$ and Erk $1 / 2$ 778 expression of H4 cells. Data were analyzed with one-way analysis of variance (ANOVA)

779 followed by Dunnett multi-comparison test. Data were presented as mean \pm standard 780 deviation and dot plot $(n=6) .{ }^{* *} p<0.01$ versus naïve control. Scale bar: $50 \mu \mathrm{m}$. NC:

781 naïve control; VC: vehicle control; P: propofol; p-Akt: phospho-Akt; Erk1/2: 782 extracellular-signal-regulated kinase 1/2; p-Erk1/2: phospho-Erk 1/2.

Figure 8. HIF-1a expression in lung cancer and neuroglioma cells after propofol administration.

786 The immunofluorescent staining with the dye of HIF-1a (green) co-stained with DAPI 787 (blue) for lung cancer A549 cells (A) and neuroglioma H4 cells (D). The representative 788 western blotting bands of HIF-1 $\alpha$ and GAPDH of lung cancer (B) and neuroglioma (E).

789 The statistical analysis of the relative ratio of HIF-1 $\alpha$ to GAPDH in A549 cells (C) and 790 H4 cells (F). Data were analyzed with one-way analysis of variance (ANOVA) followed 791 by Dunnett multi-comparison test. Data were presented as mean \pm standard deviation 792 and dot plot $(n=6) .{ }^{* *} p<0.01$ versus naïve control. Scale bar: $50 \mu m$. NC, naïve 793 control; VC, vehicle control; P, propofol, HIF-1a: hypoxia-inducible factor-1 alpha.

795 Figure 9. Propofol alters lung cancer cell mRNA expression levels of tumor 796 metastatic related genes assessed by PCR array and qRT-PCR.

797 Lung cancer A549 and neuroglioma H4 cells were treated with $4 \mu \mathrm{g} / \mathrm{mL}$ propofol or 
798 intralipid as the control for 2 hours and then recovered for up to 24 hours. A. The PCR 799 array analysis of tumor metastatic related genes. Unsupervised hierarchical cluster 800 analysis using Euclidean distance from the low-density arrays. Propofol up-regulated 8018 anti-tumor genes and down-regulated 6 pro-tumor genes of A549 cells $(n=3)$. The 802 data is relative to endogenous control, GAPDH. Red and green colors indicate 803 relatively high and low expression, respectively. The results of CXCL12 (B) and 804 CXCR4 (C) obtained from A549 cells using PCR array and qRT-PCR (D: CXCL12 and $805 \mathrm{E}: \mathrm{CXCR} 4, \mathrm{n}=3)$. The expression levels of CXCL12 gene $(\mathbf{F})$ and CXCR4 gene $(\mathbf{G})$ 806 from $\mathrm{H} 4$ cells evaluated with qRT-PCR $(n=5)$. Data were expressed as mean \pm 807 standard deviation and dot plot. ${ }^{*} p<0.05$ versus control. C: control; P: propofol;

808 VEGFA: vascular endothelial growth factor A; CTBP1: C-terminal binding protein 1;

809 CST7: cystain 7; CTSK: cathepsin K; CXCL12: C-X-C motif chemokine 12; CXCR4:

810 C-X-C chemokine receptor type 4; NR4A3: nuclear receptor subfamily 4 group A

811 member 3; RB1: retinoblastoma susceptility 1; NME1: NME/NM23 nucleoside 812 diphosphate kinase 1; MTSS1: metastasis suppressor I-BAR domain containing 1;

813 NME4: NME/NM23 nucleoside diphosphate kinase 4; SYK: spleen tyrosine kinase;

814 APC: adenomatous polyposis coli; FAT1: FAT tumor suppressor homolog 1.

815

816 Figure 10. The cellular signaling interactions in lung cancer cells after propofol

817 administration.

818 Propofol down-regulates GLUT1, which decreases glucose uptake into cell plasma. 
819 Propofol also down-regulates BRP44L expression, which converts pyruvate more to

820 lactate and less to formate and acetate or enters TCA cycle. The decreased level of

821 TCA activity inhibits the generation of intermediates for the synthesis of nucleic acids,

822 fatty acids, and carbon skeleton. The Lower intracellular glucose concentration

823 induces the secretion of PEDF. PEDF inhibits both Akt and Erk phosphorylation, which

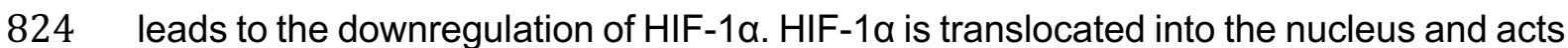

825 as a key transcriptional regulator to increase anti-tumor-related genes or decrease pro-

826 tumor-related genes. Propofol disturbs metabolism and alters tumor metastatic related

827 and ultimately inhibits the malignancy of lung cancer cells.

828 GLUT1: glucose transporter 1; BRP44L: brain protein 44-like; TCA: tricarboxylic acid;

829 PEDF: pigment epithelium-derived factor; Erk1/2: extracellular signal-regulated kinase

830 1/2; HIF-1a: hypoxia-inducible factor 1 alpha.

831

832

833

834

835

836

837

838

839 

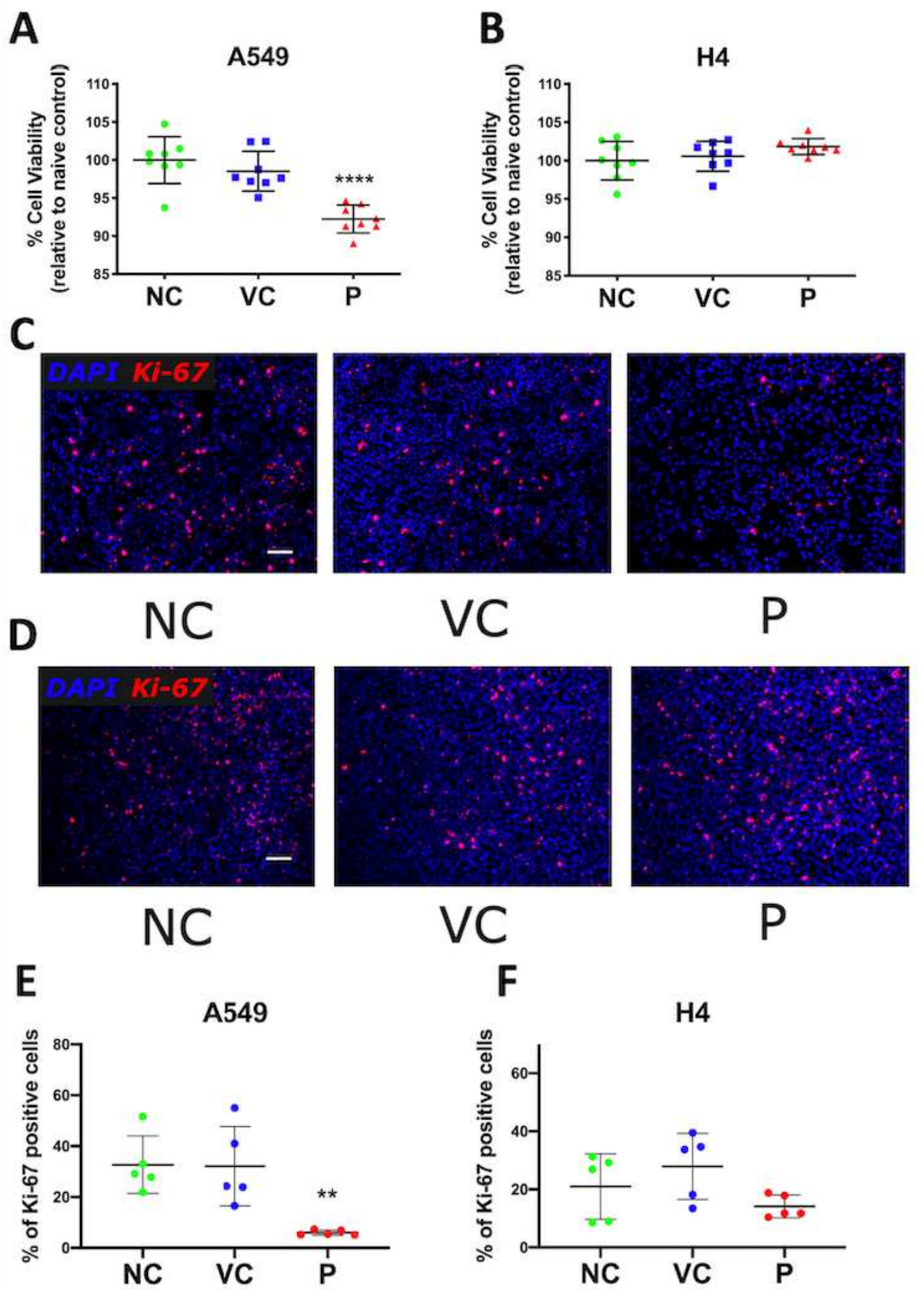

Figure 1

The cell viability and proliferative capability of lung cancer and neuroglioma cells after propofol exposure. Lung cancer A549 and neuroglioma H4 cells were treated with intralipid (vehicle control), 4 $\mu \mathrm{g} / \mathrm{mL}$ propofol, or pure culture media (naïve control). Cell viability of lung cancer $(A)$ and neuroglioma 
cells (B) was evaluated with the CCK-8 assay. Cell proliferative capability of A549 (C) and H4 cells (D) with Ki-67 immunofluorescent staining. The comparison of Ki-67 positive cell percentage in A549 cells (E) and $\mathrm{H} 4$ cells $(F)$. Data were expressed as mean \pm standard deviation and dot plot $(n=5-8)$. ${ }^{\star \star} p<0.01$, $\star \star * \star p<0.0001$ versus naïve control. Scale bar: $100 \mu \mathrm{m}$. NC, naïve control; $V C$, vehicle control; $P$, propofol.
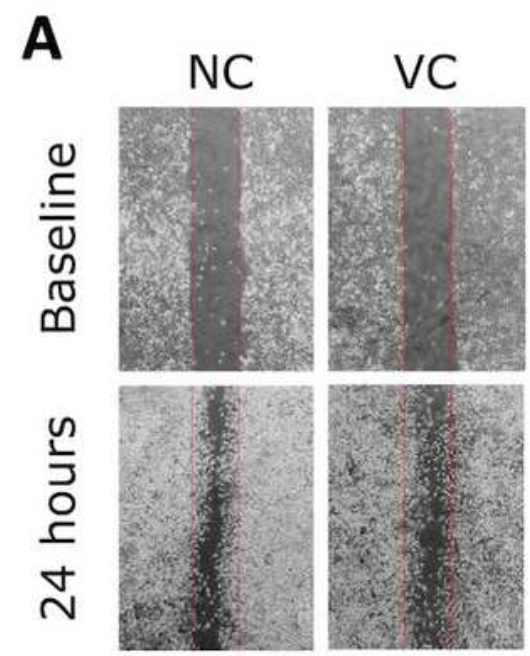

B

A549

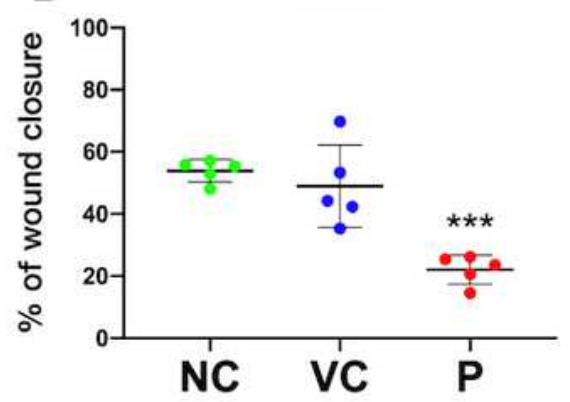

$\mathbf{E}$

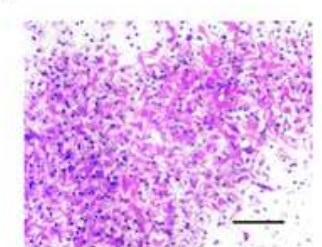

NC

G

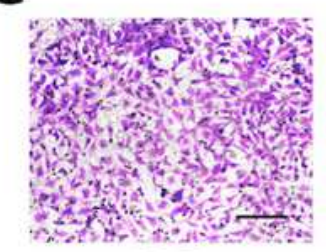

NC

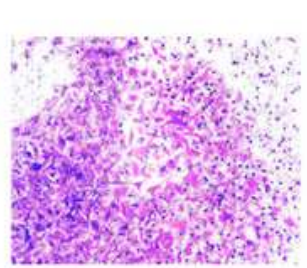

VC
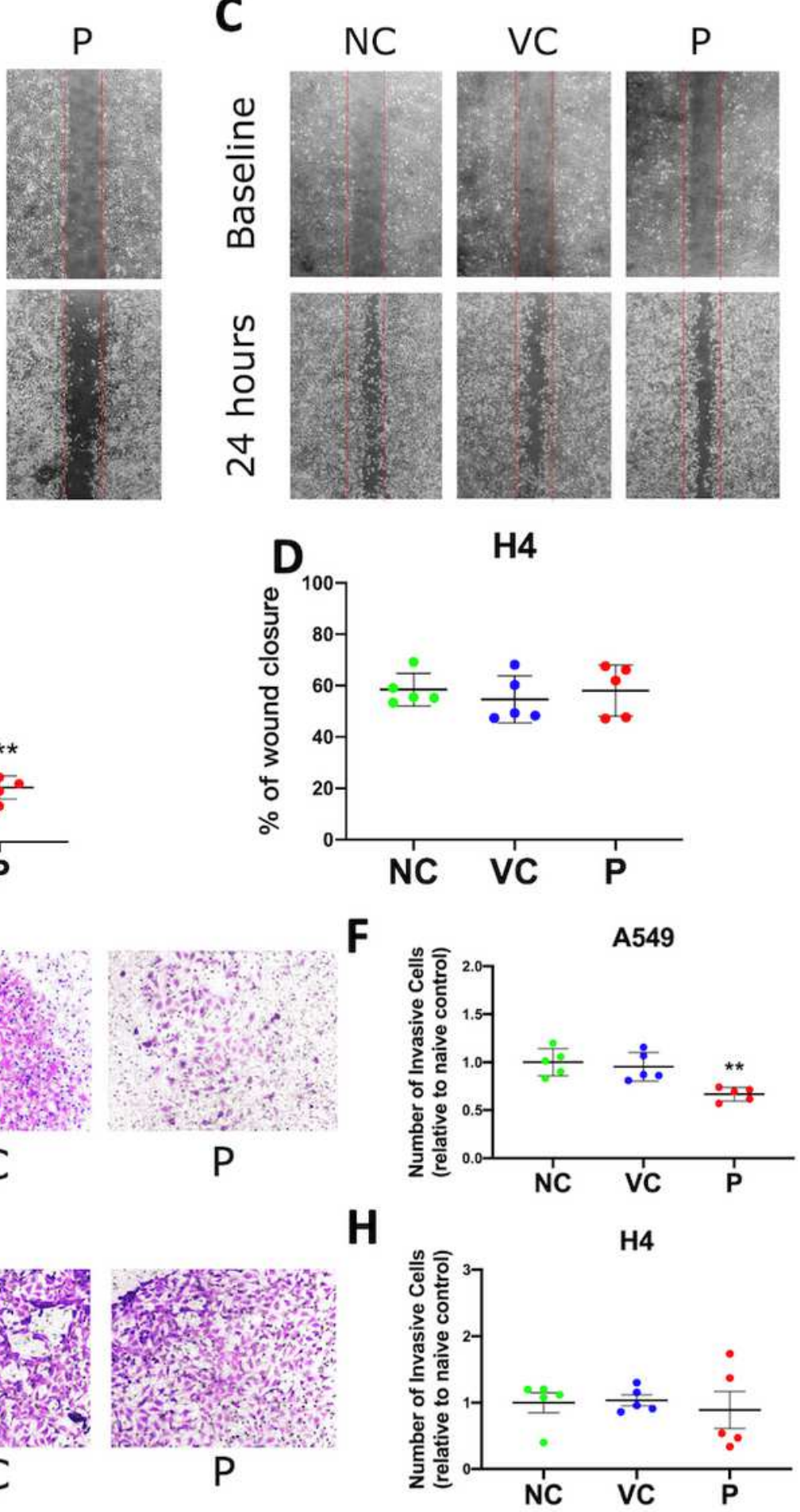

Figure 2 
The migrate and invasive ability of lung cancer and neuroglioma cells after propofol administration. Lung cancer A549 and neuroglioma H4 cells were administered with intralipid (vehicle control), $4 \mu \mathrm{g} / \mathrm{mL}$ propofol, or no treatment (naïve control). The migration of lung cancer (A) and neuroglioma cells (C) was assessed via wound healing assay with statistical analysis of the percentage of gap closure of A549 (B) and $\mathrm{H} 4$ cells (D). The invasion of A549 (E) and H4 cells (G) was evaluated by Transwell assay with the statistical analysis of the relative ratio of invasive cell number to NC in A549 (F) and H4 cells $(\mathrm{H})$. Data were presented as mean \pm standard deviation and dot plot $(n=5)$. ${ }^{\star \star} p<0.01$, ${ }^{\star \star *} p<0.001$ versus naïve control. Scale bar: $100 \mu \mathrm{m}$. NC: naïve control; V: vehicle control; P: propofol. 


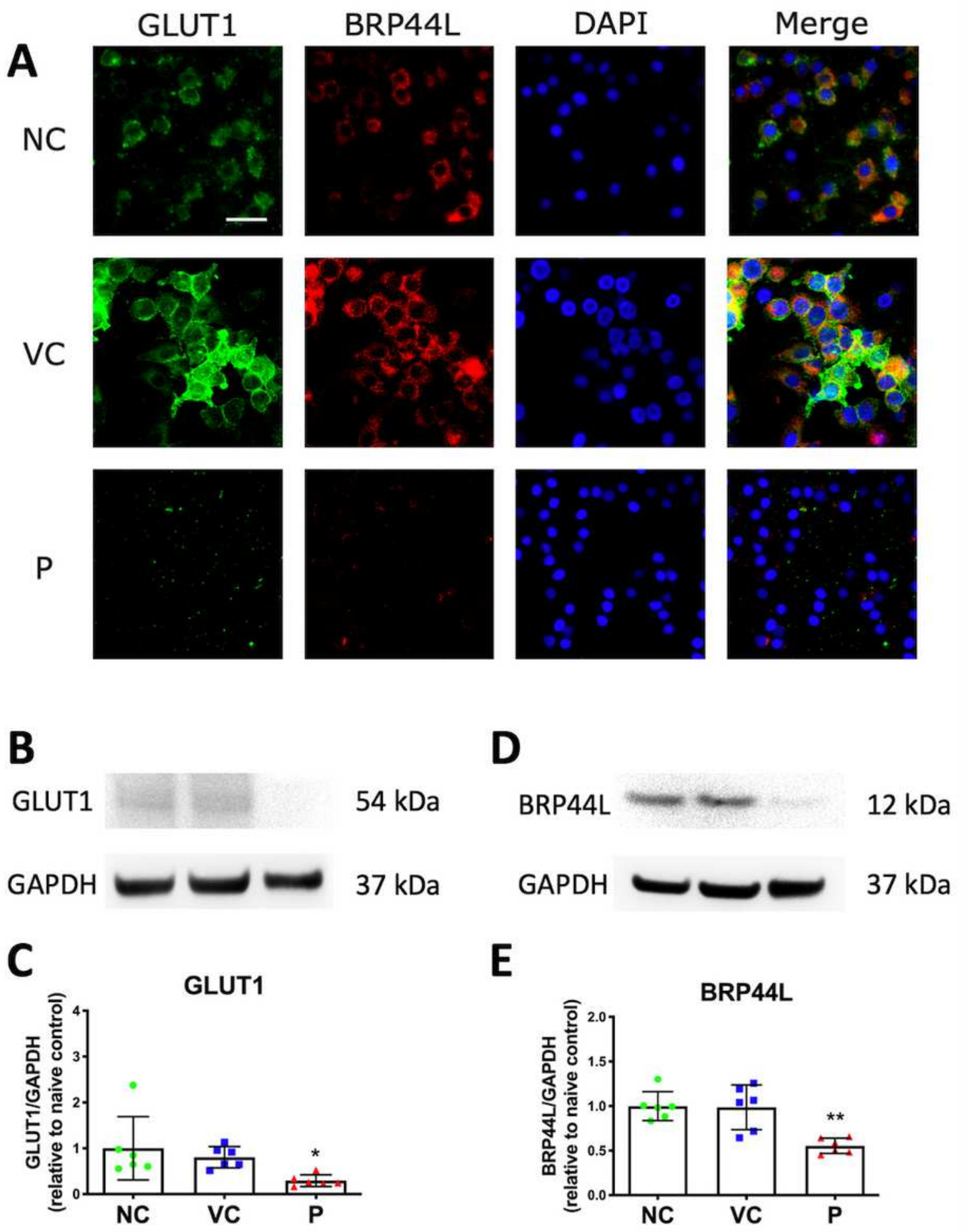

Figure 3

Propofol down-regulated GLUT1 and BRP44L in lung cancer cells. Lung cancer A549 cells were administered with $4 \mu \mathrm{g} / \mathrm{mL}$ propofol or intralipid (vehicle control) or media without drugs (naïve control) for 2 hours followed by 24 hours recovery time. GLUT1 (green) and BRP44L (red) expressions were identified by dual-immunofluorescent staining (A). The expression levels of GLUT1 (B and C) and BRP44L ( $D$ and $E$ ) were validated with western blotting analysis. The intensity of western blotting bands was 
normalized by housekeeping gene GAPDH. Data were analyzed with one-way analysis of variance (ANOVA) followed by Dunnett multi-comparison test. Data were presented as mean \pm standard deviation and dot plot $(n=6) .{ }^{\star} p<0.05 ;{ }^{\star \star} p<0.01$ versus naïve control. Scale bar: $50 \mu \mathrm{m}$. NC: naïve control; VC: vehicle control; P: propofol; GLUT1: glucose transporter 1; BRP44L: brain protein 44- like.
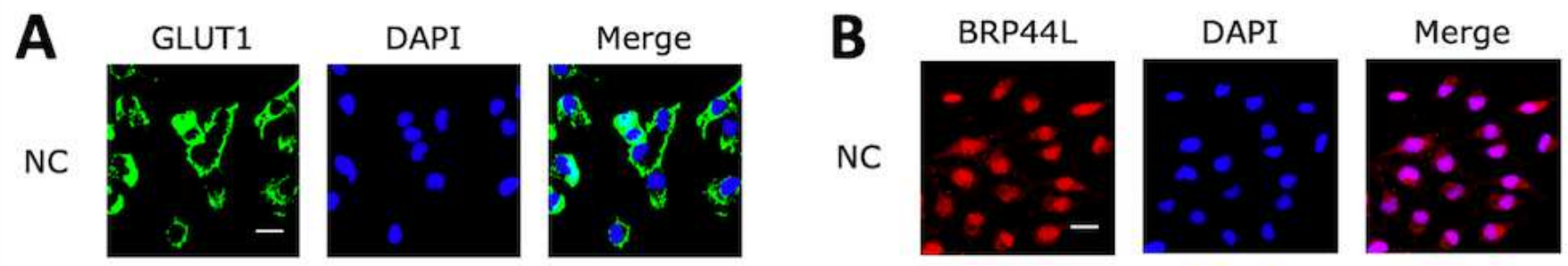

vc
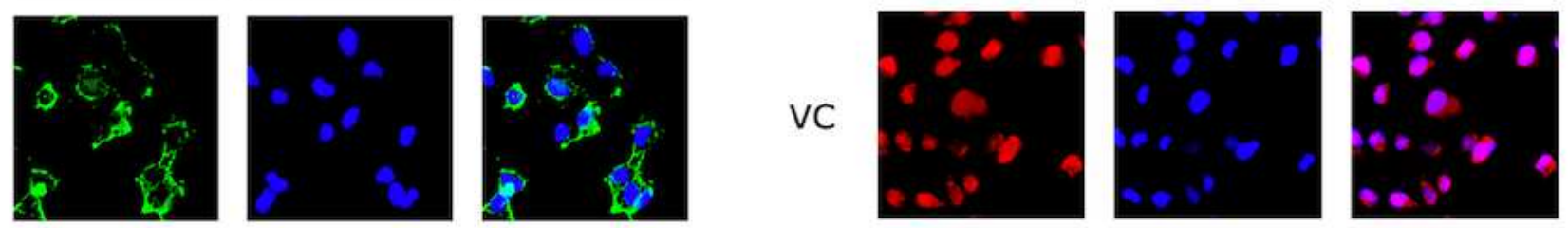

$\mathrm{P}$
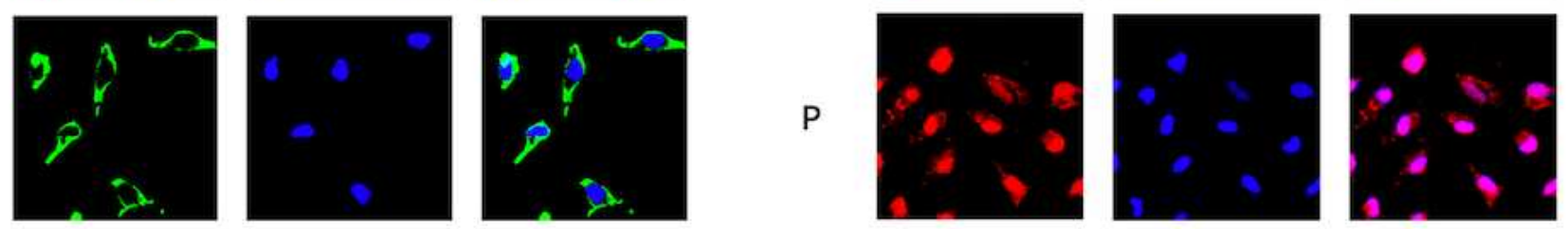

C

GLUT1

$54 \mathrm{kDa}$

$\mathbf{E}$
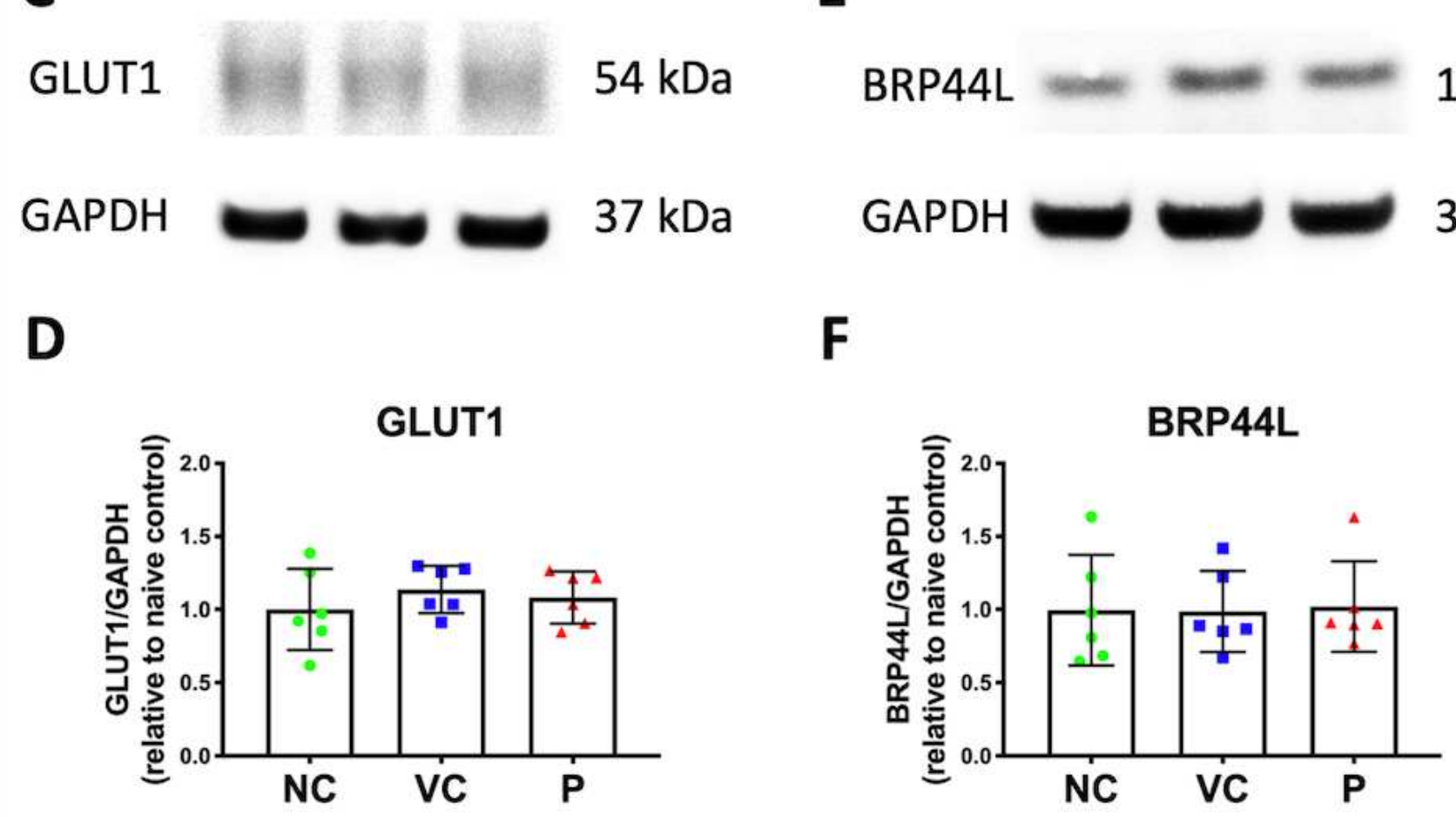

Figure 4 
GLUT1 and BRP44L expressions in neuroglioma cells after administered with propofol. Neuroglioma H4 cells were administered with a clinically relevant concentration of $4 \mu \mathrm{g} / \mathrm{mL}$ propofol. GLUT1 (green) and BRP44L (red) expressions were analyzed by immunofluorescent staining ( $A$ and B) and validated with western blotting (C-F). The intensity of Western blot bands was normalized by housekeeping gene GAPDH. Data were analyzed with one-way analysis of variance (ANOVA) followed by Dunnett multicomparison test. Data were presented as mean \pm standard deviation and dot plot $(n=6)$. Scale bar: $50 \mu \mathrm{m}$. NC: naïve control; VC: vehicle control; P: propofol; GLUT1: glucose transporter 1; BRP44L: brain protein 44-like.
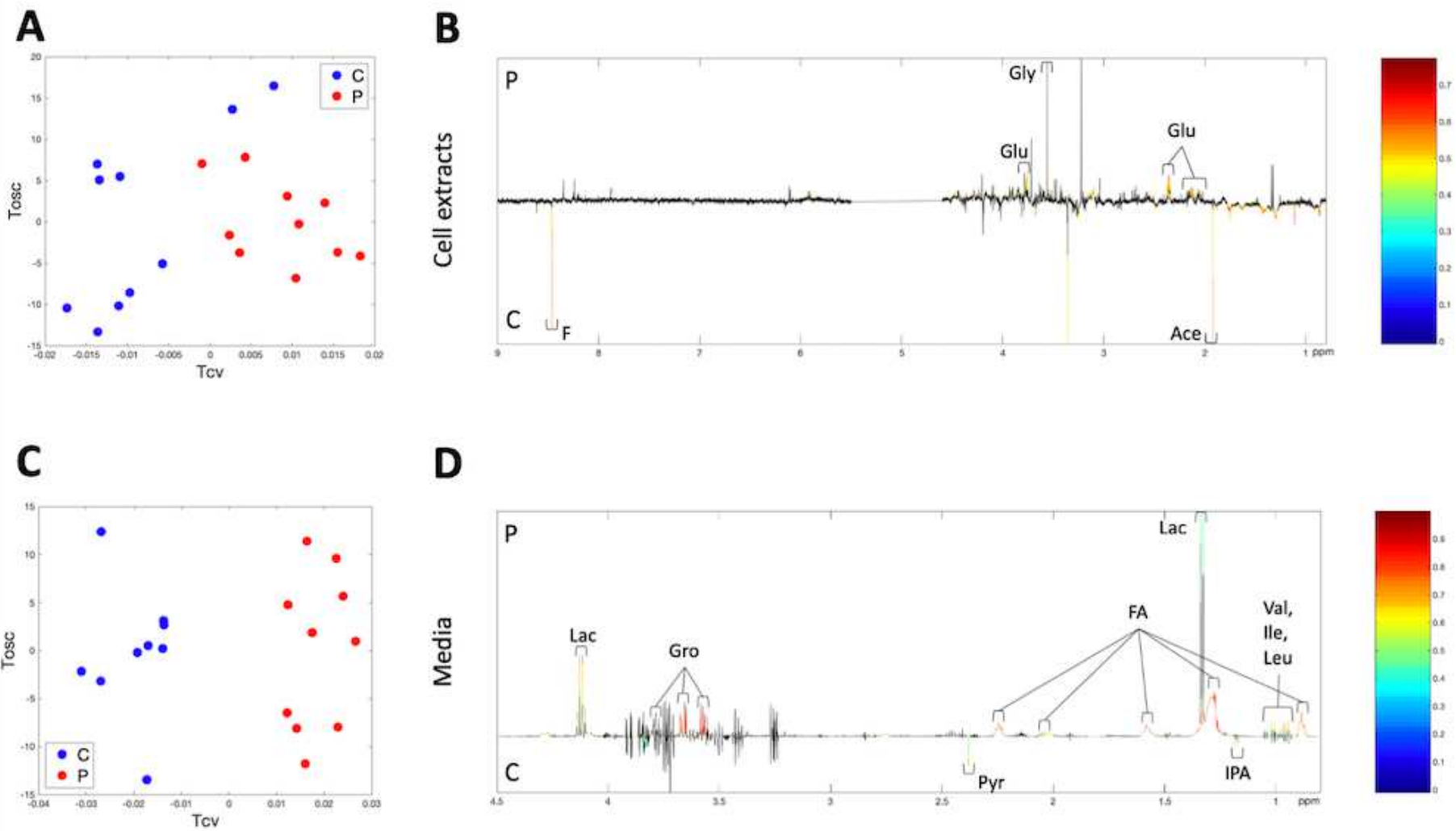

D
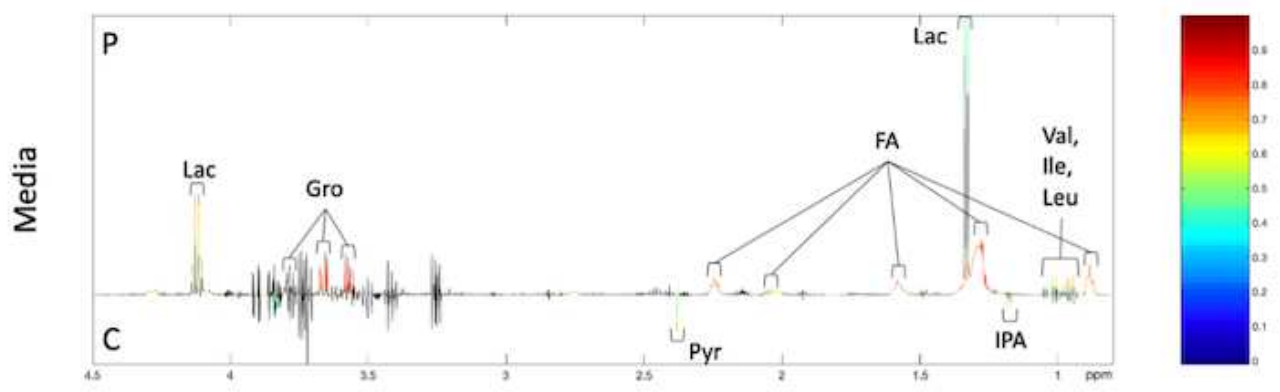

\section{Figure 5}

The metabolism alterations of lung cancer cells after propofol administration. Orthogonal projection to latent structures-discriminant analysis (OPLS-DA) loadings plots from $1 \mathrm{H}$ NMR spectral data of the A549 cell extract $(A$ and $B)$ and $A 549$ media samples $(C$ and $D)$ for comparisons as control vs. propofol $(n=9-$ 10). A and C: OPLSDA scores plot; B and D: OPLS-DA loadings plot. The color bar indicates the correlation coefficient values ( $r 2$ ) to be high in red and low in blue. C: control; P: propofol; F: formate; Glu: glutamate; Gly: glycine; Ace: acetate; Lac: lactate; Gro: glycerol; Pyr: pyruvate; FA: fatty acids; IPA: isopropanol; Val: valine; lle: isoleucine; Leu: leucine. 

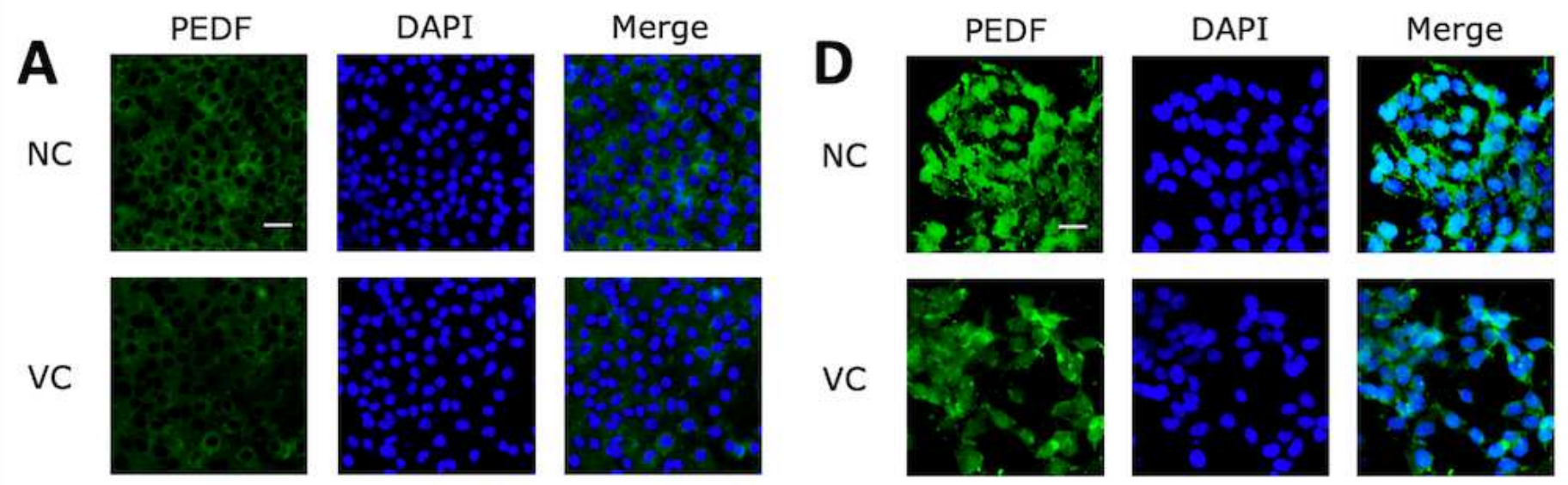

P
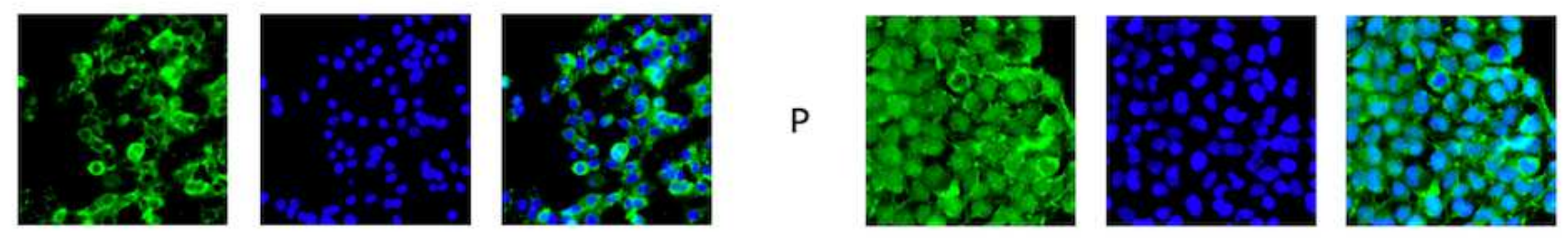

B

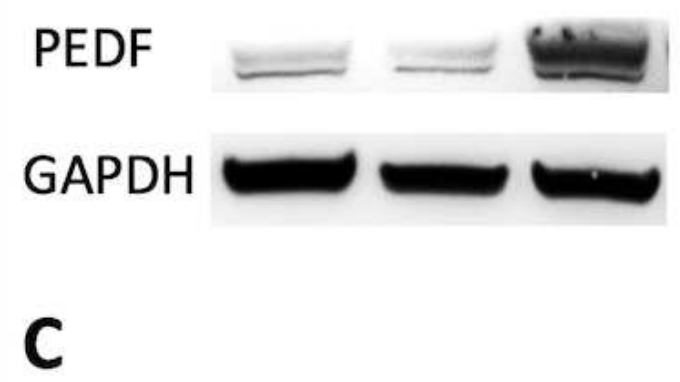

$46 \mathrm{kDa}$

\section{$37 \mathrm{kDa}$}

$\mathbf{F}$

$\mathbf{E}$
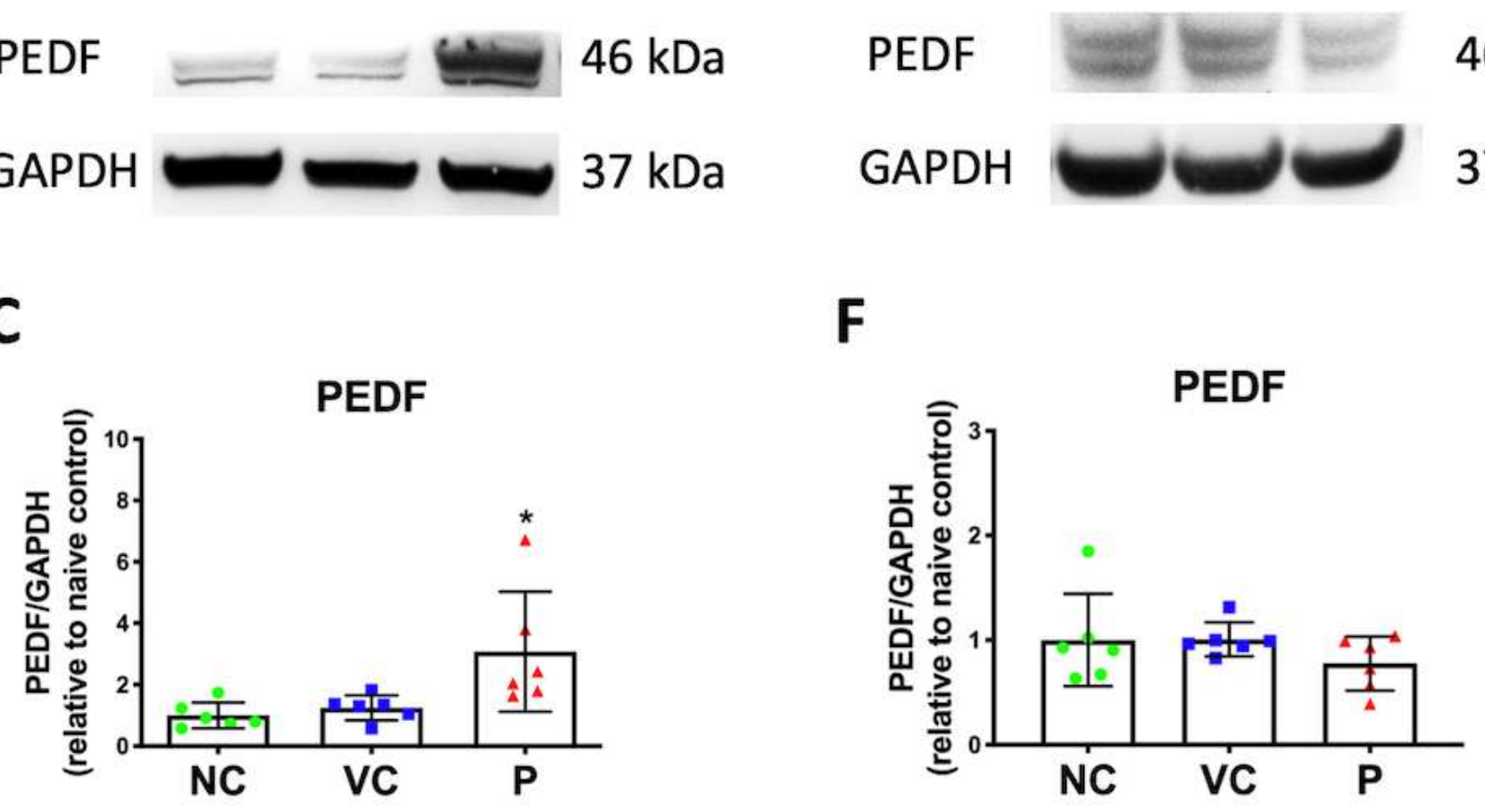

$46 \mathrm{kDa}$

37 kDa

\section{Figure 6}

Propofol up-regulated PEDF in lung cancer cells but not neuroglioma cells. Lung cancer A549 and neuroglioma $\mathrm{H} 4$ cells were administered with a clinically relevant concentration $4 \mu \mathrm{g} / \mathrm{mL}$ of propofol for 2 hours. PEDF expression was analyzed by immunofluorescent staining (A. A549 cells and D. H4 cells) and western blotting analysis (B. A549 cells and E. H4 cells). PEDF (green) was overlaid with DAPI (blue). The intensity of western blotting bands was normalized by housekeeping gene GAPDH (C. A549 cells and F. 
A549 cells). Data were analyzed with one-way analysis of variance (ANOVA) followed by Dunnett multicomparison test. Data were presented as mean \pm standard deviation and dot plot $(n=6)$. ${ }^{*}<0.05$ versus naïve control. Scale bar: $50 \mu \mathrm{m}$. NC, naïve control; VC, vehicle control; P, propofol; PEDF; pigment epithelium-derived factor.
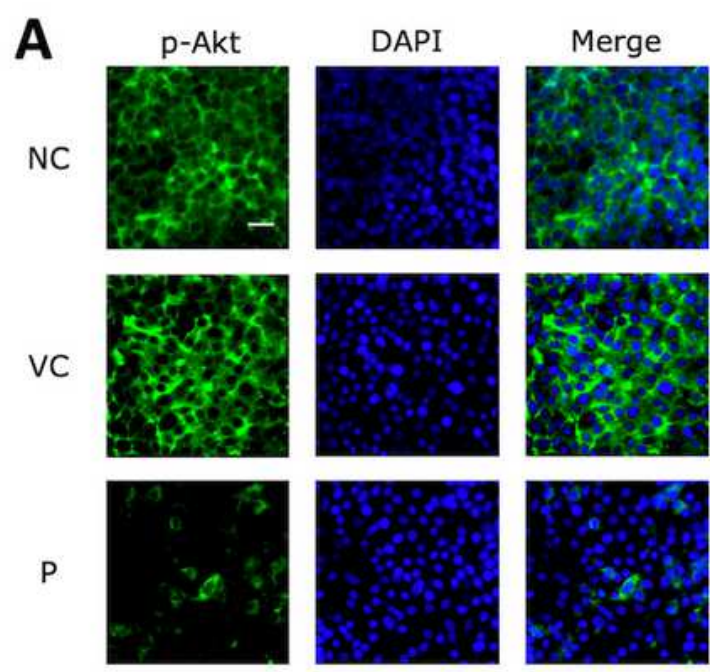

C

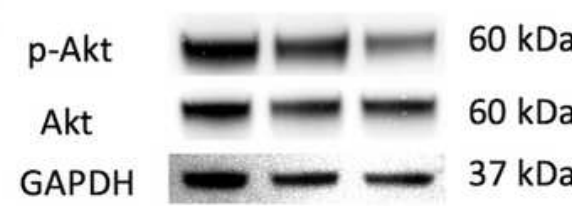

D

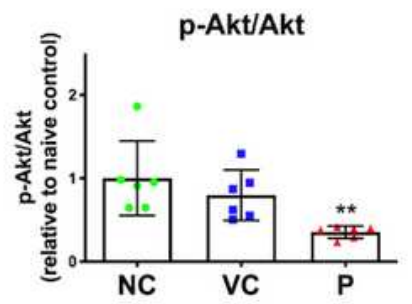

G

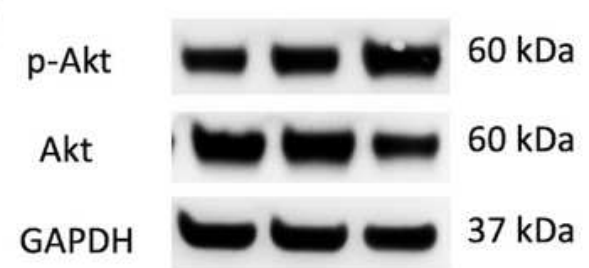

H

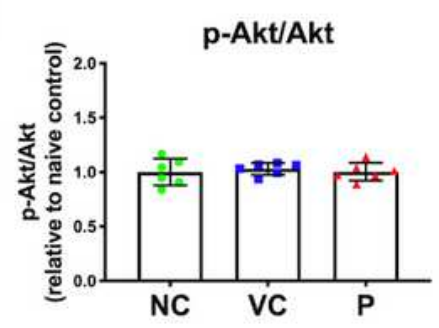

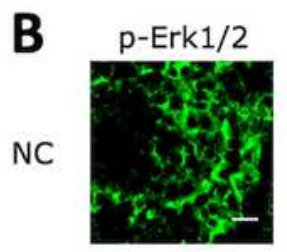
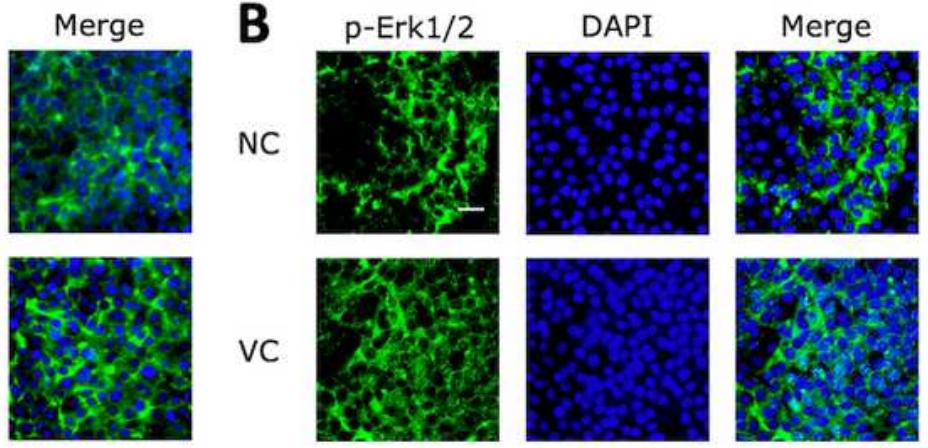

VC
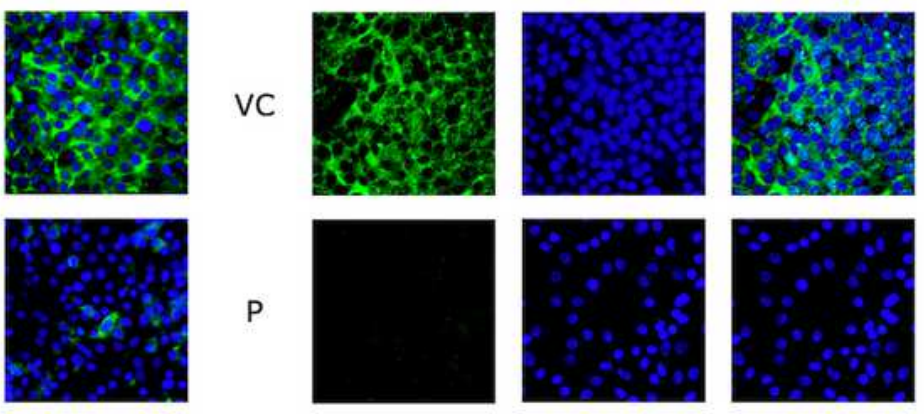

$\mathbf{E}$

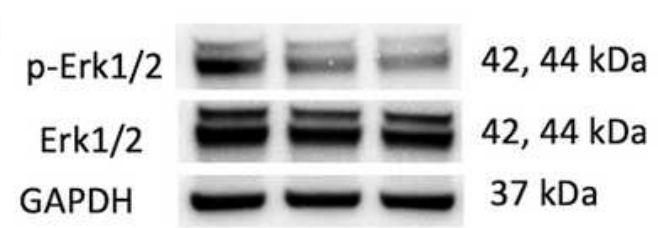

F

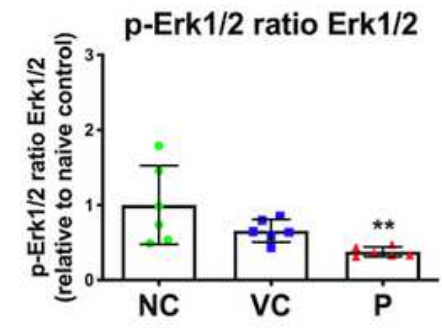

I

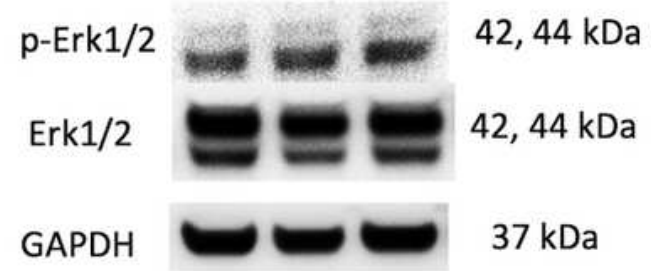

J

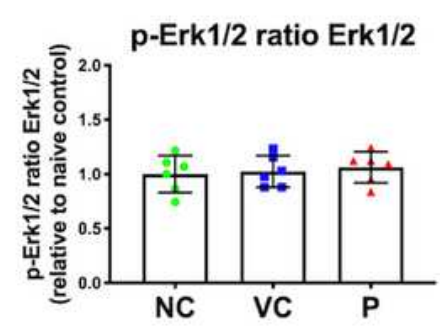

Figure 7 
Propofol down-regulated Akt and Erk expression in lung cancer cells but not neuroglioma cells. Lung cancer A549 and neuroglioma $\mathrm{H} 4$ cells were treated with $4 \mu \mathrm{g} / \mathrm{mL}$ propofol or vehicle control or naïve control. A. p-Akt (green) of lung cancer cells as cytoplasmic dyeing overlaid with DAPI (blue) staining in nucleus. B. p-Erk1/2 (green) was stained in cytoplasm overlaid with DAPI (blue) in the nucleus. C. The representative western blotting bands of p-Akt and Akt expression of A549 cells. D. The relative ratio of pAkt to Akt compared to naïve control of A549 cells. E. The representative bands of $p$-Erk1/2 and Erk1/2 expression of $A 549$ cells. F. The representative western blotting bands of $p$ - Erk1/2 and Erk1/2 expression of A549 cells. G. The representative bands of $\mathrm{p}$-Akt and Akt expression of $\mathrm{H} 4 \mathrm{cells}$. $\mathrm{H}$. The relative ratio of p-Akt to Akt compared to naïve control of $\mathrm{H} 4$ cells. I. The representative bands of $p$-Erk1/2 and Erk $1 / 2$ expression of $\mathrm{H} 4$ cells. J. The representative western blotting bands of $\mathrm{p}$-Erk $1 / 2$ and Erk $1 / 2$ expression of $\mathrm{H} 4$ cells. Data were analyzed with one-way analysis of variance (ANOVA) followed by Dunnett multicomparison test. Data were presented as mean \pm standard deviation and dot plot $(n=6)$. ${ }^{*} p<0.01$ versus naïve control. Scale bar: $50 \mu \mathrm{m}$. NC: naïve control; VC: vehicle control; P: propofol; p-Akt: phosphoAkt; Erk1/2: extracellular-signal-regulated kinase 1/2; p-Erk1/2: phospho-Erk 1/2. 

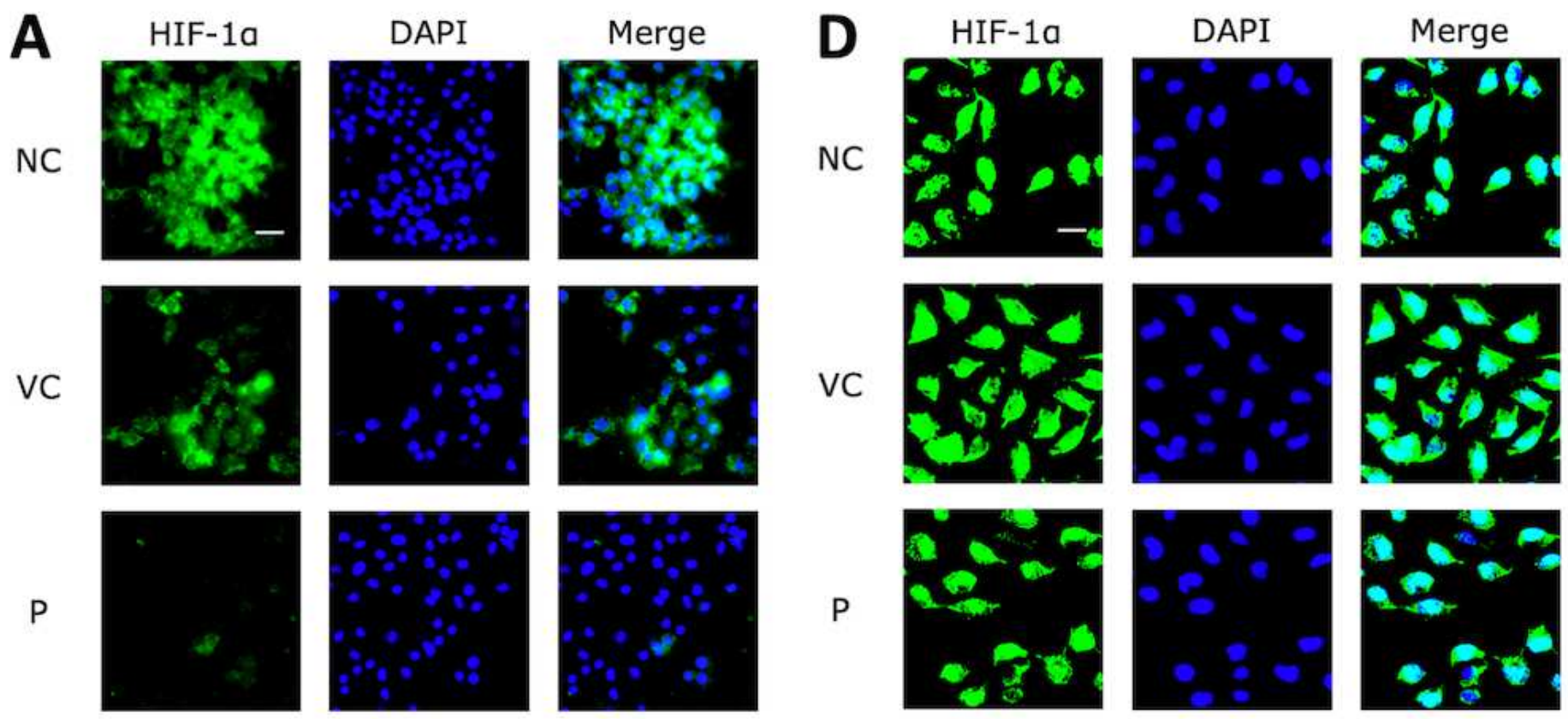

B

E

HIF-1 $\alpha$

$93 \mathrm{kDa}$

HIF-1 $\alpha$

$93 \mathrm{kDa}$

\section{GAPDH}

37 kDa

GAPDH

37 kDa

C

F
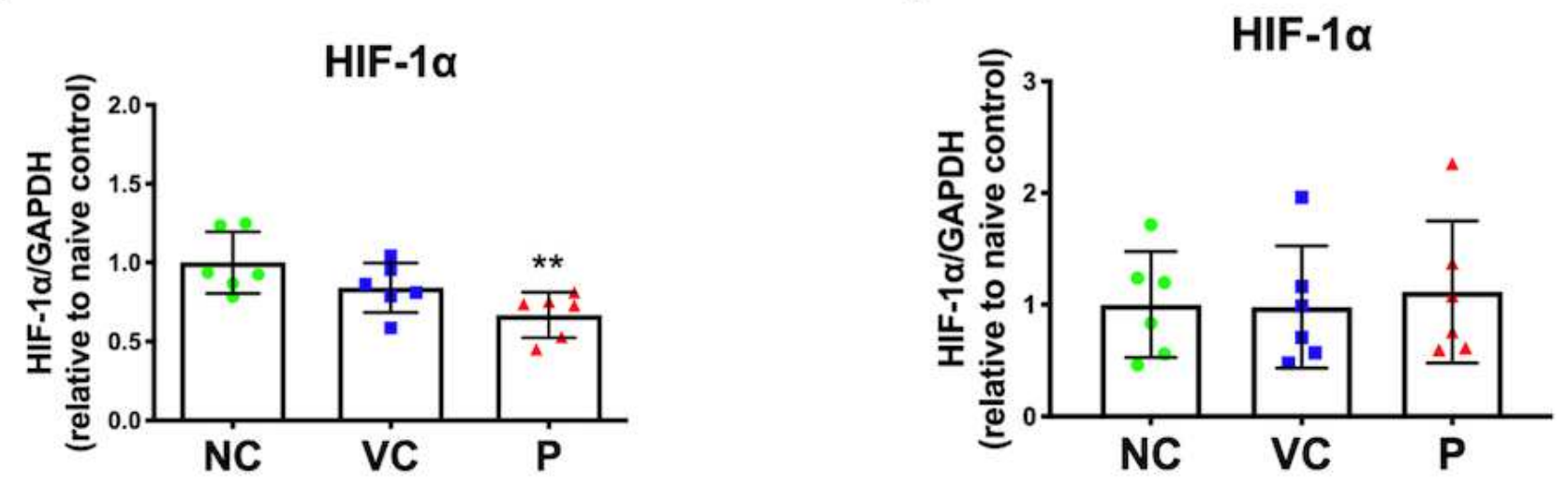

Figure 8

HIF-1a expression in lung cancer and neuroglioma cells after propofol administration. The immunofluorescent staining with the dye of HIF-1a (green) co-stained with DAPI (blue) for lung cancer A549 cells (A) and neuroglioma H4 cells (D). The representative western blotting bands of HIF-1 $\mathrm{a}$ and GAPDH of lung cancer (B) and neuroglioma (E). The statistical analysis of the relative ratio of HIF-1a to GAPDH in A549 cells (C) and H4 cells (F). Data were analyzed with one-way analysis of variance 
(ANOVA) followed by Dunnett multi-comparison test. Data were presented as mean \pm standard deviation and dot plot $(n=6) .{ }^{* \star} p<0.01$ versus naïve control. Scale bar: $50 \mu \mathrm{m}$. NC, naïve control; VC, vehicle control; P, propofol, HIF-1a: hypoxia-inducible factor-1 alpha.
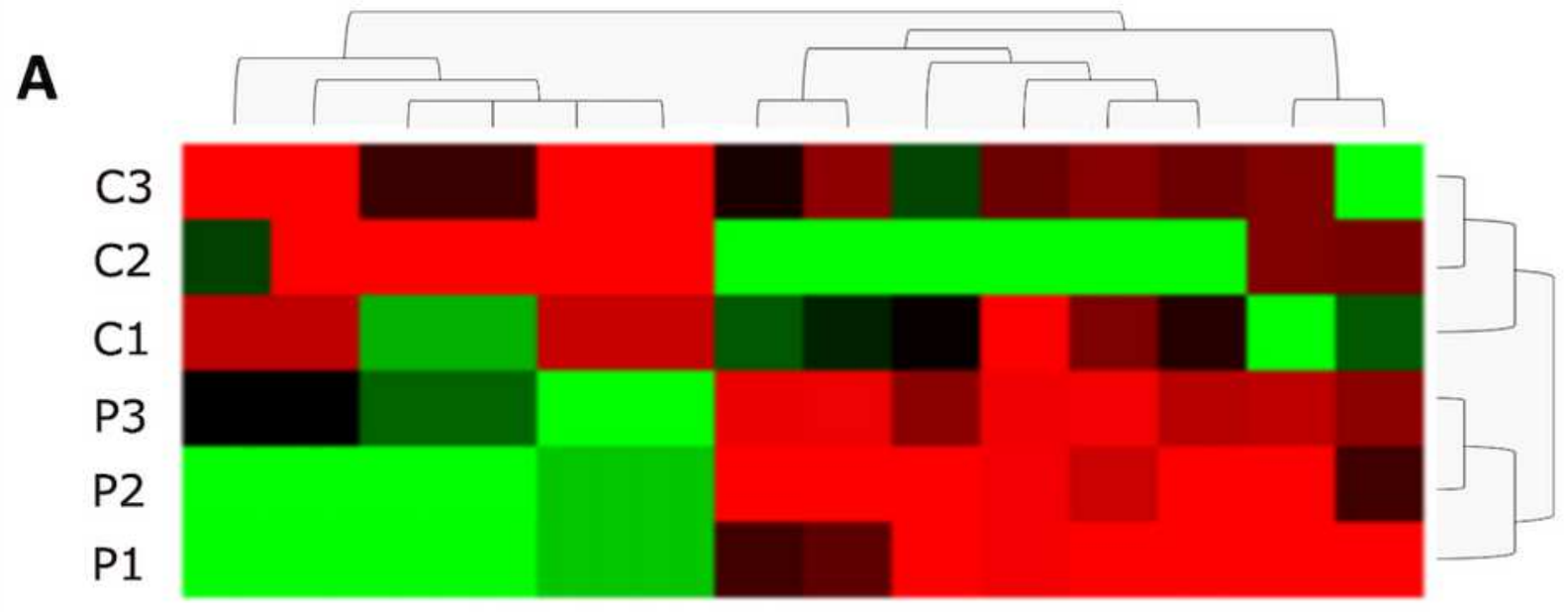

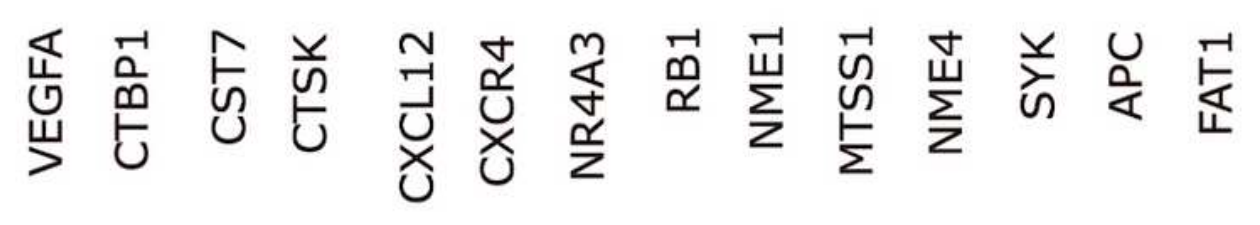

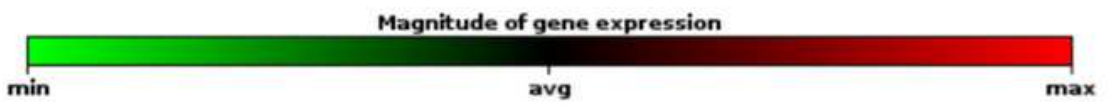

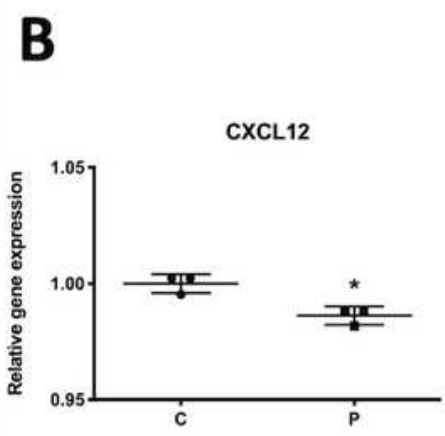
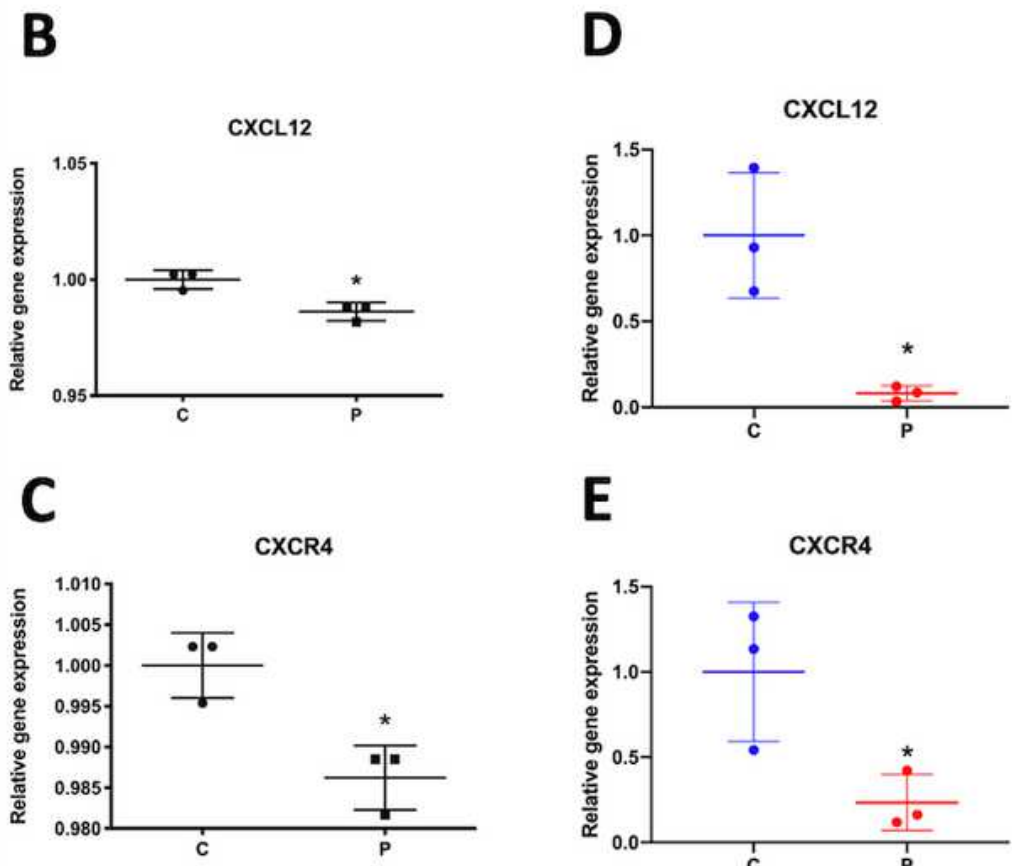

E

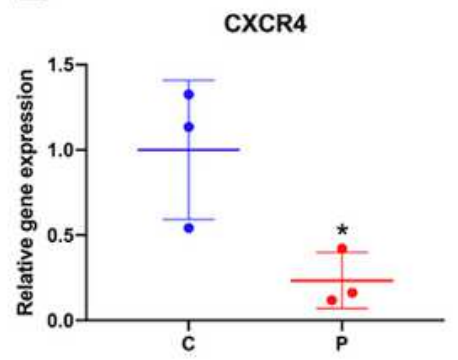

$\mathbf{F}$
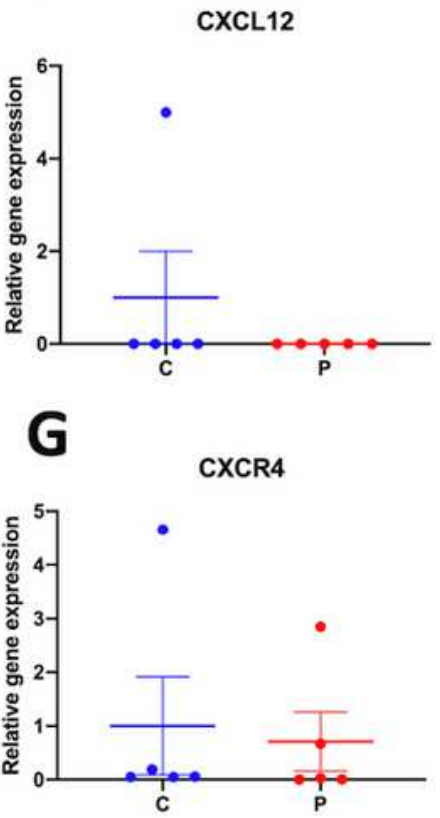

Figure 9

Propofol alters lung cancer cell mRNA expression levels of tumor metastatic related genes assessed by PCR array and qRT-PCR. Lung cancer A549 and neuroglioma H4 cells were treated with $4 \mu \mathrm{g} / \mathrm{mL}$ propofol 
or intralipid as the control for 2 hours and then recovered for up to 24 hours. A. The PCR array analysis of tumor metastatic related genes. Unsupervised hierarchical cluster analysis using Euclidean distance from the low-density arrays. Propofol up-regulated 8 anti-tumor genes and down-regulated 6 pro-tumor genes of A549 cells $(n=3)$. The data is relative to endogenous control, GAPDH. Red and green colors indicate relatively high and low expression, respectively. The results of CXCL12 (B) and CXCR4 (C) obtained from A549 cells using PCR array and qRT-PCR (D: CXCL12 and E: CXCR4, $n=3)$. The expression levels of CXCL12 gene $(F)$ and CXCR4 gene $(G)$ from H4 cells evaluated with qRT-PCR $(n=5)$. Data were expressed as mean \pm standard deviation and dot plot. ${ }^{*} p<0.05$ versus control. C: control; P: propofol; VEGFA: vascular endothelial growth factor A; CTBP1: C-terminal binding protein 1; CST7: cystain 7; CTSK: cathepsin K; CXCL12: C-X-C motif chemokine 12; CXCR4: C-X-C chemokine receptor type 4; NR4A3: nuclear receptor subfamily 4 group A member 3; RB1: retinoblastoma susceptility 1; NME1: NME/NM23 nucleoside diphosphate kinase 1; MTSS1: metastasis suppressor I-BAR domain containing 1; NME4: NME/NM23 nucleoside diphosphate kinase 4; SYK: spleen tyrosine kinase; APC: adenomatous polyposis coli; FAT1: FAT tumor suppressor homolog 1. 


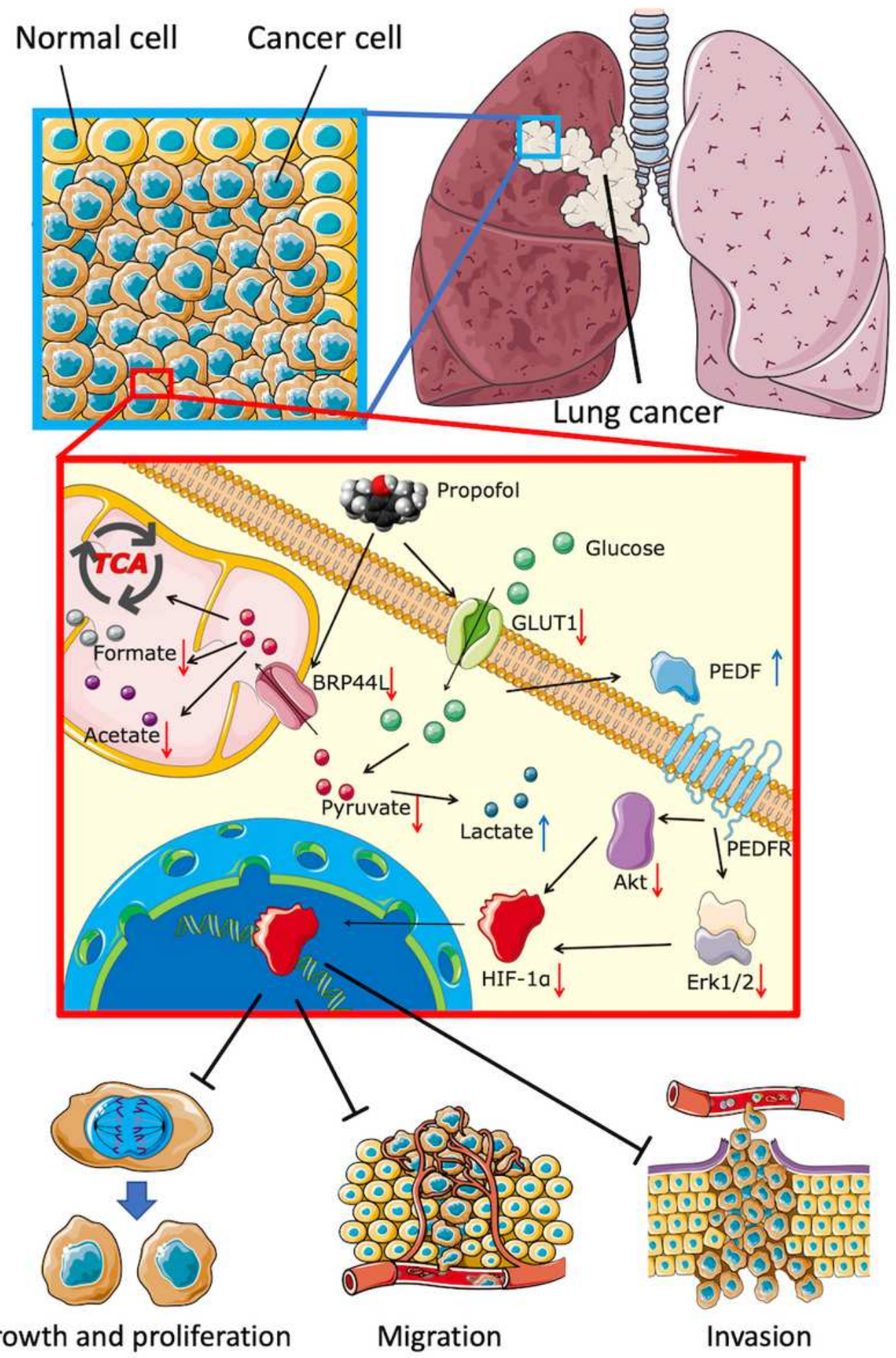

Figure 10

The cellular signaling interactions in lung cancer cells after propofol administration. Propofol downregulates GLUT1, which decreases glucose uptake into cell plasma. Propofol also down-regulates BRP44L expression, which converts pyruvate more to lactate and less to formate and acetate or enters TCA cycle. The decreased level of TCA activity inhibits the generation of intermediates for the synthesis of nucleic acids, fatty acids, and carbon skeleton. The Lower intracellular glucose concentration induces 
the secretion of PEDF. PEDF inhibits both Akt and Erk phosphorylation, which leads to the downregulation of HIF-1a. HIF-1a is translocated into the nucleus and acts as a key transcriptional regulator to increase anti-tumor-related genes or decrease protumor- related genes. Propofol disturbs metabolism and alters tumor metastatic related and ultimately inhibits the malignancy of lung cancer cells. GLUT1: glucose transporter 1; BRP44L: brain protein 44-like; TCA: tricarboxylic acid; PEDF: pigment epithelium-derived factor; Erk1/2: extracellular signal-regulated kinase 1/2; HIF-1a: hypoxia-inducible factor 1 alpha.

\section{Supplementary Files}

This is a list of supplementary files associated with this preprint. Click to download.

- Supplements.docx

- Supplements.docx 